\title{
4 Die Entstehung der Idee der Annales Ecclesiastici
}

Seit der Drucklegung des ersten Bandes der Magdeburger Zenturien stellte die Kirchenhistoriographie ein zentrales Arbeitsgebiet für die römische Kurie dar. Die Entwicklungen seit der Papstwahl Pius' IV. bis zum Antritt Gregors XIII. haben gezeigt, dass die Kirchengeschichte keineswegs durch einen privaten und in sich geschlossenen Beichtkreis wie den Filippo Neris beansprucht werden konnte. Selbst der Jesuitenordern, der sich an den Arbeiten der ständig einberufenen Congregatio Germanica beteiligte, nahm sich dieser Disziplin nur in begrenztem Masse an. Für die Jesuiten war Kirchengeschichte nur insofern von Bedeutung, als dass sie ausschliesslich in Hinblick auf ihr Potenzial zum Zweck der Ordensentwicklung im Bereich der doctrina christiana und der Entstehung einer einheitlichen Ratio studiorum für die Bildungsinstitutionen des Ordens angesehen wurde. ${ }^{469}$ Diese Entwicklungen fordern eine Neubewertung des Entstehungsprozesses der Annales Ecclesiastici des Oratorianer Cesare Baronio: Wie konnten die Annales überhaupt zu Stande kommen, wenn die Kirchenhistoriographie ausschliesslich ein zentraler Aufgabenbereich innerhalb der römischen Kurie war und sich damit keineswegs mit den Interessen des Oratoriums in San Giovanni dei Fiorentini überschnitt? Die Antwort auf diese Frage soll im Folgenden zeigen, dass Baronios Annales weit mehr ein bewusstes Produkt der römischen Kurie waren, als es die Forschung bislang wahrnehmen wollte.

\subsection{Der kirchenhistoriographische Beitrag der Correctores Romani}

Nam quis nescit primam esse historiae legem, ne quid falsi dicere audeat? Deinde ne quid veri non audeat? ${ }^{470}$

Der Entstehungsprozess einer neuen Kirchengeschichte, die aber nicht weiter als Historia Ecclesiastica betitelt wurde, überschneidet sich mit dem grossen Unternehmen der Correctores Romani, das Decretum Gratiani zu überarbeiten und neu zu publizieren. Diese Arbeiten wurden mit dem Breve Cum pro munere pastorali Gregors XIII., in welchem die editio Romana der Dekretensammlung 1582 bestätigt wurde, abgeschlossen. Das grossangelegte Unterfangen der Correctores Romani, dessen Anfänge bis zum Pontifikat Pius' IV. zurückreichen, sah die Festlegung eines authentischen Textes für das gesamte Corpus Iuris Canonici vor. ${ }^{471}$ Erst im Ghislieri-Pontifikat Pius' V. wurde 1566 dafür eine eigenständige Kommission eingesetzt, die ursprünglich aus fünf Kardinälen

469 O’Malley 1993, S. 225-242; Testa 2002, S. 524-538.

470 Cicero, De Oratore, II, XV, 62.

471 Das Breve Cum pro munere pastorali Gregors XIII. ist abgedruckt in Corp. iur. can., I, S. LXXIXLXXXII.

Ә Open Access. () 2022 Filip Malesevic, publiziert von De Gruyter. (c) BY-NC-ND Dieses Werk ist lizenziert unter einer Creative Commons Namensnennung - Nicht-kommerziell - Keine Bearbeitung 4.0 International Lizenz. https://doi.org/10.1515/9783110741117-005 
und fünf Doktoren der Rechte bestand. Guglielmo Sirleto, Francesco Alciati, Ugo Boncompagni, Marcantonio Colonna und Alessandro Sforza sind als Kardinäle aufgelistet, während in der Auszählung der doctores der spanische Theologe Francisco Torres auftaucht. ${ }^{472}$ Der Jesuitentheologe Torres brachte 1572 seine Adversus Magdeburgenses Centuriatores erstmals heraus. Das Werk entstand aus einem produktiven Austausch zwischen den Revisionsarbeiten der Correctores Romani am Decretum Gratiani und den kurialen Widerlegungsarbeiten gegen die Magdeburger Zenturiatoren. Damit liefen die Widerlegungsversuche, die bislang aus der Entstehungs- und Entwicklungsgeschichte der Congregatio Germanica und der der Indexkongregation erklärt wurden, auf die Kirchenkanonistik über. Bereits 1563 gab der Gräzist und Kontroverstheologe Francisco Torres, der als päpstlicher Theologe an der letzten Sitzungsperiode des Tridentinums teilnahm, eine Edition der Apostolischen Konstitutionen heraus. 1578 gab er diese Edition, die er mit einer reich kommentierten und in Latein verfassten Übersetzung erweiterte, neu heraus. Diese rechtlich-liturgische Textsammlung der Alten Kirche stellte für Torres den Inbegriff der apostolischen Lehre dar. Die Apostolischen Konstitutionen enthalten nämlich die „nichtschriftliche apostolische Tradition“, weshalb sie seitens der Protestanten zurückgewiesen wurden. ${ }^{473}$ Torres versuchte in seiner Edition, die Konstitutionen Papst Clemens I. zuzuschreiben und in das erste Jahrhundert zu datieren. Dabei stellte er einen deutlichen Mangel am historisch-kritischen Sinn dieser Zuschreibung fest. Deshalb erweist sich dieser Jesuitentheologe im Zusammenhang mit der Entstehung von Baronios Annales Ecclesiastici als interessante Figur, die jedoch seitens der Forschung bislang kaum berücksichtigt wurde. Die Arbeiten Francisco Torres' und sein Gesamtwerk zeigen, dass der spanische Jesuitentheologe an der kurialen Widerlegung der Zenturiatoren und ihrer Ecclesiastica Historia beteiligt war. Seine Edition der Apostolischen Konstitutionen, in deren Einleitung er die Bedeutung der Tradition anhand philosophischer Argumente erläutert, enthält unter anderem auch einen Elenchus dogmatum haereticorum. In diesem zählt Torres über hundert Lehrpunkte auf und widerlegt damit die einzelnen theologischen Positionen der Protestanten mit Hilfe der Apostolischen Konstitutionen. Die Mehrzahl dieser Lehrpunkte leitete Torres aus der bereits genannten Confessio Augustiniana, die als Referenzwerk für die Widerlegung der Confessio Augustana galt und welche sein Bruder Hieronymus zusammengestellt hatte, ab. Der Elenchus enthält Lehrpunkte, die sich sowohl auf die Magdeburger Zenturien als auch auf den römischen Messritus beziehen. Die Forschung

472 BAV, Vat. lat. 4889, f. 4r-5r. Vgl. auch Theiner, Disquisitiones criticae, App. I, Nr. 3-4; Corp. iur. canon., S. LXXVI-LXXVII.

473 Torres, Constitutiones Sanctorum Apostolorum, S. 6: „Scripsit igitur hos libros Clemens ex persona Apostolorum, sicut epistulam ad Corinthios ex persona ecclesiae Romanae. Quid enim interest ex persone ecclesiae Romanae ad Corinthios scripsisse valde utilem epistulam [...] an scripsisse ex persona Apostolorum ad universam ecclesiam christianae et ecclesiasticae disciplinae Constitutiones?“ Baronio merkte in seinen Annales an, dass ihm dieses Werk in drei Handschriften vorlag (Ann. Ecc., II, S. 17-24). 
hatte sich bislang diesem Elenchus im Hinblick auf die Widerlegung der Zenturien nicht gewidmet, weshalb dieser nun erstmals im Zusammenhang mit Torres` Adversus Magdeburgenses untersucht werden soll.

Die Widerlegung der Magdeburger Zenturien hing von einem theologisch-dogmatischen Verständnis darüber, wie eine authentisch korrekte Kirchengeschichte mit der Autorität des römischen Messritus zusammenhing, ab. Mit seinem Elenchus dogmatum haereticorum ergänzte Torres diesen Zusammenhang um ein weiteres Element, das die Bedeutung des Bischofsamtes betrifft. Die von Papst Clemens I. zusammengestellten Konstitutionen enthalten einen Teil, in dem spezifisch die Hierarchie der Kirchenämter angesprochen wird. Aus diesem Teil leitete Torres den Lehrsatz gegen die Theologie der Lutheraner ab, dass sich sowohl in der Heiligen Schrift als auch in den Psalmen bereits eine apostolisch-ursprüngliche Form der Messzelebration finden lasse. Diesen Lehrsatz hatte ebenfalls Heinrich Bullinger in seinen Schriften vertreten. Die ursprüngliche Form des römischen Messritus muss sodann optimam sein, denn die Messefeier weist dadurch eine besondere Nähe sowohl zur Predigt über die Menschwerdung Gottes als auch zum eigentlichen Ritus des Brotbrechens auf. Der apostolische Ursprung einer rituellen Begründung der Messe und ihrer Feier sollte außerdem auch schon deshalb gewährleistet werden, da sie mit der Gottesverehrung eine Einheit darstellte. ${ }^{474}$ Francisco Torres hatte diese Einheit zwischen Messritus und Gottesverehrung bereits während der Debatten um die Residenzpflicht der Bischöfe am Tridentinum verteidigt. Torres war ein eifriger Verteidiger der Position, die die Residenzpflicht als ius divinum ansah. Der Streit um die bischöfliche Residenzpflicht kam deshalb in der dritten Sitzungsperiode des Tridentinums wieder auf, da 113 Bischöfe das am 13. Januar 1547 verabschiedete Dekret zur Residenzpflicht nicht befolgten. Nach fast zehn Jahren befanden sich diese Bischöfe nicht in ihren Diözesen, sondern in Rom. Um die Residenzpflicht als rechtsgültig und damit dem Kirchenrecht entsprechend zu erklären, musste sie als ius divinum begründet werden. ${ }^{475}$ In diesem Zusammenhang gab Francisco Torres bereits 1551 einen Traktat zur Residenzpflicht heraus. Sein De residentia pastorum

474 Torres, Constistutiones Sanctorum Apostolorum, II, S. 39 (obs. 1): „Huius Apostolicae doctrinae testimonio convincitur, non laudare Lutheranos Deum neque venerari, siquidem de sublimibus doctrinae Christianae articulis inter se contendunt [...] ex Confessionistis in thesibus suis faretur \& restatur, \& alij alios inter se pro haereticis habent ut Confessionistes [...] \& adiaphoristas, ac principes Saxoniae [...] sectas Lutheranorum tanquam execrabiles condemnarunt [...]“. Die observatio bezieht sich auf die Stelle in ibid., c. 38-39: ,[...] sicut in caelo \& in tessa, ut sicut caelites naturae incorporeae cuncti Deum uno consensu laudant, sic etiam in terra cuncti homines uno ore, \& uno animo, concelebrent solum Deum unum, \& verum per Christum unigenitum. Voluntas itaque Dei est, ut ipsum idem sentiendo laudemus, idemque dicendo veneremur“. Hinsichtlich Bullingers theologischen Geschichtsdenken vgl. insbes. Garcia Achilla 1992.

475 Für eine Zusammenfassung der bischöflichen Residenzpflicht während der ersten Sitzungsperiode vgl. Jedin 1966, Bd. 2, S. 399-404; O’Malley 2013, S. 112-113, 116-118. Zum ersten Dekret über die Residenzpflicht vgl. dann COD, S. 681-682 sowie CT, v, S. 847-848, 871-872. 
iure divino scripto sancita stellt eine Lösung zum Problem bereit, wie das ius divinum $\mathrm{zu}$ begründen war und in welchem Zusammenhang es mit dem Amt des Bischofs stand. Torres verstand das Bischofsamt als eine von Gott verliehene Würde. Der Papst, der den Bischof seiner Diözese zuteilt, übe im Ritus der Bischofsweihe daher nur die Funktion eines minister aus. Ferner besitze der Bischof durch die Berufung des Petrus - „Weide meine Schafe“ (Jh. 21, 17) - grundsätzlich dieselbe Binde- und Lösegewalt wie die Päpste, auch wenn ihm diese Gewalt der Papst verleiht. ${ }^{476}$ Die zu ihrer letzten Sitzung versammelten Konzilsteilnehmer erklärten somit die Residenzpflicht zum göttlichen Recht, weil sich das bisherige Dekret nur auf die Androhung von Strafen beschränkt hatte. Jedoch waren sich die Konzilsväter darüber uneinig, ob die Residenzpflicht überhaupt entscheidend für das Bischofsamt war. Genauso gut konnte die Residenzpflicht nur als Vorgabe des kanonischen Rechtes auslegt werden. ${ }^{477}$ Die Debatten um die Residenzpflicht sollten auch nachhaltig das bereits diskutierte OrdoDekret beeinflussen, denn mit ihr wurde die grundsätzliche Problematik, ob die Bischöfe ihre Amtsgewalt bereits durch ihren Weiheritus von Gott erhielten oder ob ihnen diese Gewalt erst durch ihre Ernennung durch den Papst zugesprochen wurde, angesprochen. Die Lösung dieses Problems hatte weitreichende ekklesiologische Konsequenzen in Hinblick darauf, wie das Bischofsamt im Kirchenkörper nicht nur gemäss dem Kirchenrecht verstanden werden sollte.

Der Streit um die bischöfliche Residenzpflicht kann anhand der theologischen Positionen der beiden Fraktionen rekonstruiert werden, die sich um den Erzbischof von Ragusa, Ludovico Beccadelli, und den Kardinallegaten Ludovico Simonetta gebildet hatten. ${ }^{478}$ Es ist aber entscheidend, die zentralen Passagen des Reformdekrets von der 23. Sitzung im Juli 1563 festzuhalten, da in diesem die Residenzpflicht nicht als ius divinum, sondern lediglich als praeceptum divinum - als „göttliche Weisung“ also - deklariert wurde:

Da durch göttliche Weisung allen, denen die Seelsorge anvertraut ist, geboten ist, ihre Schafe zu kennen, für sie das Opfer darzubringen, sie durch die Verkündigung des Wortes Gottes, durch die Verwaltung der Sakramente und durch das Beispiel aller guten Werke zu weiden, wie ein Vater für die Armen und die anderen Elenden Sorge zu tragen und sich den übrigen pastoralen Aufgaben zu widmen [...] ermahnt sie die hochheilige Synode eindringlich, der göt-

476 Torres, De residentia, S. 21-22: „[...] a Deo fieri singulos episcopos, a quo fuerit institutus episcopatus, una Dei voce, unaque lege de pascendis ovibus ad omnes rectores et magistors ecclesiarum per Petrum transeunte, quem ipse Dominus absque ullo hominum ministerio, neque ab hominibus, necque per hominem episcopum primum, ceterorum episcoporum ac pastorum omnium principem et gubernatorem fecit, voce illa, atque lege de pascendis ovibus ei singulariter sive specialiter dicta, per quem ceteros pastores et rectores animarum facit [...] Per se enim pontifex, id est, suo nomine, \& virtute nullam gratiam, neque charisma ministrare potest, sed ex virtute, quam Deus dat, sicut B. Petrus ait“.

477 Jedin 1949-1975, Bd. 4/1, S. 114-115; idem 1966, Bd. 2, S. 91-97; Fragnito 2010, S. 53-78.

478 O’Malley 2013, S. 180-181, 199-200, 217-219; Wiesner 2016, S. 221-254. 
tlichen Gebote eingedenk zu sein und die Herde als ihr Vorbild in Gerechtigkeit und Wahrheit $\mathrm{zu}$ weiden und $\mathrm{zu}$ führen. ${ }^{479}$

Es kann nicht die Rede davon sein, dass sich die Konzilsväter in Trient während ihrer Arbeit an diesem Dekret um eine eigenständige Ekklesiologie bemühten. Der Konzilspräsident Girolamo Seripando legte nämlich selbst ein Schema über die Residenzpflicht vor. Außerdem formulierte er die Bitte, die Frage nach dem ius divinum nicht anzusprechen, sondern dafür zu sorgen, dass die Bischöfe tatsächlich in ihren Diözesen residierten. ${ }^{480}$ Das Bischofsamt steht für die Verschmelzung der Verkündigung der Menschwerdung Gottes mit der Verwaltung der Sakramente, woraus Francisco Torres dann seine Verteidigung des römischen Messritus ableitete. Das protestantische Schisma entstand Torres zufolge aus einer bewussten Ablehnung dieser im Bischofsamt enthaltenen Einheit und der im Sakrament des ordo eingeschriebenen Geschlossenheit zwischen Predigt und Sakramentsverwaltung. Demgemäss stellen die Magdeburger Zenturien für den Jesuitentheologen eindeutig das Resultat einer Beeinträchtigung der in den Apostolischen Konstitutionen zentral verankerten vita communis dar, die sich aus dem Verhältnis zwischen Diözesanbischof und seiner Weide ergibt. Dieses für die Kirche sinnstiftende Verhältnis wird durch die beneficentiae der Bischöfe gegenüber ihrer Herde geschaffen, da das Bischofsamt auch die Gewalt des Sündenerlasses enthält. ${ }^{481}$ Die Zenturiatoren brachten somit ein Werk heraus, mit welchem diese sakramentale Ordnung des Kirchenkörpers grundsätzlich gestört wurde. Dem Gläubigen wird dadurch die Hoffnung auf die Sündenvergebung als die wohl wichtigste Bindung zu Gott selbst verwehrt.

479 Zit. n. Wohlmuth 2000-2002, Bd. 3, S. 744. Vgl. hierzu die entsprechende Passage in COD, S. 744. Zentral für die Favorisierung dieser Wortverwendung seitens Pius' IV. vgl. insbes. seine Briefkorrespondenz mit dem Konzilslegaten Simonetta in Šusta, 1909-1914, II, S. 89, 92, 127-131, in welchen Simonetta die Sachlage dadurch verzerrte, dass er sie dem Papst so präsentierte, als ob sich die Befürworter des ius divinum gegen die Kurie und damit Rom verschwören würden. Vgl. so auch die Briefschreiben Pius' IV. an die Konzilslegaten vom 11. Mai 1562, in denen er den Legaten seine Unzufriedenheit kundtut: in ibid., S. 132-143. Hierzu dann auch O’Malley 2013, S. 181, der zutreffend diese verzerrte Darstellung Simonettas als kuriale Angst vor den „ghosts of Constance and Basel” erklärt. Vgl. auch das Urteil des Franziskanertheologen Francisco de Córdoba in CT, XIII/2, S. 275: „[...] sunt duo concilia in ecclesia: nam est unum concilium congregatum Tridenti et aliud concilium congregatum Romae cum papa. Ista duo concilia aliquo modo ex opposito pugnant“. Weitere Meinungen der Theologen in ibid., S. 262-285.

480 CT, IX, S. 133-134. Dieses Schema verfasste der spanische Kanonist Antonio Augustín zusammen mit Paleotti und Musotti, Seripandos Sekretär. Für eine Abschrift vgl. CT, IX, S. 135-137 sowie den Eintrag in Paleottis Tagebuch in ibid., III/1, S. 461-462.

481 Torres, Constitutiones Sanctorum Apostolorum, II, S. 98. Zu Torres‘ Meinung über die Tridentiner Residenzpflicht vgl. seine im Mai verfassten Gutachten an Seripando und Hosius in BAV, Barb. lat. 817, f. 229v-233v, 233v-236v, in denen er das Verständnis der Residenzpflicht als remedium kritisierte. Vgl. ebenfalls dann auch seinen eigenen Entwurf zum Dekret aus der dritten Sitzungsperiode in BAV, Barb. lat. 817, f. 225r-226r. 
Ebenso wie schon bei seiner Überarbeitung der Apostolischen Konstitutionen möchte Francisco Torres auch in seinen Adversus Magdeburgenses Centuriatores die historische Kontinuität der Constitutiones aufzeigen. Ein zentrales Schriftstück aus der Biblioteca Vaticana, das zu einem angemessenen Verständnis dieses Traktats beiträgt, bietet die in der Handschrift des Jesuitentheologen verfasste De ratione scribendi adversus centurias ecclesiasticae historiae. Darin zählt Torres die drei Pfeiler auf, auf denen eine Widerlegung der Magdeburger Zenturien stattfinden sollte: Glaubenslehre (doctrina), Zeremonialwesen (caeremoniis) und Kirchenverwaltung (ecclesiastica politica). ${ }^{482}$ Torres stellte damit einen strategischen Plan zu einer gezielten Widerlegung der Zenturien vor. Zusätzlich fragte er danach, wie diese drei Bereiche miteinander zusammenhängen. Der Jesuitentheologe Torres ist somit nicht an einzelnen Widerlegungsbereichen, die vor ihm bereits andere Autoren geleistet haben, interessiert. Vielmehr möchte er nachweisen, wie sich diese drei Bereiche in der Kirchengeschichtsschreibung ,wunderbar“ (mirifice) bestätigen lassen. ${ }^{483}$ Bislang wurde dieses dreiteilige Schema einer kirchenhistoriographischen Widerlegung der Magdeburger Zenturien seitens der Forschung nicht genügend auf seinen theologischen Gehalt hin untersucht.

In der Widerlegungsstrategie des spanischen Jesuiten Francisco Torres tauchen zum ersten Mal die Kirchenzeremonien und ihre zentrale Bedeutung für die Kirchenhistoriographie auf. Genauso wie die Glaubenslehre und die Kirchenverwaltung mittels der Geschichte in den göttlichen Heilsplan verortet werden, so sind auch die damit verbundenen Zeremonien und Riten als ein zentraler Baustein innerhalb der Kirchenordnung anzusehen. Luther hatte aber anhand des „Wachstums des Gotteswortes“ beobachtet, dass damit auch die Macht und der Einfluss Satans wachsen würden. Für Luther war es deshalb fragwürdig, ob denn in der Kirchengeschichte die Sicherheit der Gläubigen überhaupt gewährleistet werden konnte. Dieses Geschichtsverständnis beruht auf der Auffassung, dass die Kirchengeschichte einen ständigen Kampf zwischen dem Gotteswort und der Versuchungsmacht Satans darstellt. ${ }^{484}$ Dieselbe Auffassung konnte Francisco Torres in den Zenturien wiedererkennen. Die drei von ihm genannten Bereiche einer einheitlichen Widerlegung der Zenturien sollten

482 BAV, Vat. lat. 12608, f. 321r-322r. Orella y Unzué 1976, S. 539-541, hat eine Transkription vorgelegt, in welcher aber weder auf den theologischen Charakter noch auf den Zusammenhang mit den Adversus Magdeburgenses Centuriatores eingegangen wurde.

483 BAV, Vat. lat. 12608, f. 321r. Die Adversus Magdeburgenses Centuriatores knüpfen an das 1569 in Dillingen herausgegebene Werk De hierarchis ordinationibus an, was deren terminus post quem bestimmt. Es kann sich bei den Adversus keineswegs um den im bereits erwähnten Brief Da Mulas an Hosius von 1568 präsentierten Vorschlag handeln. Zu einer Rekonstruktion der Entstehung dieses Werkes vgl. insbes. folgende Briefkorrespondenz in Braunsberger, Canisius Epistulae et atca, VI, S. 278, 314, 334, 345, 367 und diejenige innerhalb des Jesuitenordens zwischen Nadal und Borja in MHSI, Epistulae Hieronymo Nadal, III, S. 569, 576, 580, 592, 599, 616, 632. Vgl. hierzu dann auch Orella y Unzue 1976, S. 241-243.

484 WA 15, 32, 7; ibid. 18, S. 626, 26. Vgl. zur Rezeption bei Flacius Krumwiede 1952, S. 77-79; Dörries 1961, S. 86-103; Wriedt 1996, S. 31-45; Bollbuck 2014, S. 72. 
dem Beispiel der Historia Tripartita Basilius` d. Gr. folgen. ${ }^{485}$ Matthias Flacius hatte bereits in seiner 1549 erschienenen Standfestigkeit Basilij die Legende des Bischofs und Heiligen Basilius ediert. In dieser wird berichtet, dass Kaiser Valens (328-378) die arianische Lehre, welche die Trinität und Wesensgleichheit Christi leugnete, als selbständigen Glauben anerkannte. Basilius weigerte sich jedoch diese Lehre anzunehmen, obwohl ihm der Kaiser mit Kerker und Tod drohte. Demgemäss deutete Flacius die Historia Tripartita als Begründung eines erfolgreichen Widerstands gegen staatliche Anordnungen in Glaubensfragen. ${ }^{486}$ Buch und Druck stellten für Flacius sodann die zentralen Kommunikationskanäle zur Verbreitung des Gotteswortes dar, denn es sei die Aufgabe der Geistlichen, ihr Publikum über ihre Predigten anzusprechen. Mit der Veröffentlichung solcher Predigten im Druck könnten sie aber ein noch grösseres Publikum erreichen, wodurch dann auch die Kirchenhistoriographie zu einer Art Auslegung der Offenbarungslehre werde. ${ }^{487}$

In seiner Schrift gegen die Magdeburger Zenturiatoren betont der Jesuitentheologe Francisco Torres ebenfalls den Beitrag, den das Zeremonialwesen für ein theologisch angemessenes Geschichtsverständnis darstellt. Die Zenturiatoren hätten Riten und Zeremonien als einen Kanon aufgefasst, der an die Artikel einer wahren Glaubenslehre anknüpfe, weshalb der Ausgangspunkt für deren Beschreibung in der Kontroverse um die Adiaphora zu suchen sei. Torres stellte in seinem Werk indessen einen Versuch vor, wie das Kirchenzeremoniell und dessen Riten als stetige Weiterentwicklung der Apostolischen Konstitutionen verstanden werden könnten. Torres zufolge stellen die Apostolischen Konstitutionen somit den eigentlichen Ausgangspunkt dafür da, Zeremonien kirchenhistoriographisch zu fassen. Das verbum Dei könne sich dadurch „unverdorben“ (in-corruptus) weiterentwickeln. ${ }^{488}$ Zeremonien und Riten sind somit keineswegs dem Bereich der Adiaphora zuzuordnen. Im Zusammenhang mit den umstrittenen Konzessionen des Laienkelches und der Priesterehe wurde dieser Umstand aber seitens der Kurie selbst beeinträchtigt, da keine eigentliche Rechtsgrundlage gefunden werden konnte, mit deren Hilfe diese Zugeständnisse eindeutig abgestritten werden konnten. In allen fünf Büchern der Adversus Magdeburgenses Centuriatores tauchen daher Bereiche des Zeremoniells

485 BAV, Vat. lat. 12608, f. 321v.

486 Falcius, Standhaftigkeit, c. Aiijr, Aiiijr-v. Die Historia Tripartita findet sich in PL, Bd. 69, Sp. 1093A/B, 1095C-1097A. Zur Legende selbst vgl. RGG, Bd. 2 (1999), S. 1653.

487 Ibid., c. Aijr. Zu dieser „medialen Performativität“ protestantischer Kirchengeschichtsschreibung vgl. Sandl 2000, S. 188-189; idem 2011, S. 416.

488 Torres, Adversus Magdeburgenses Centuriatores, S. 3v. Zur Abhandlung von Riten und Zeremonien in den Magdeburger Zenturien vgl. Ecc. Hist., IV, 406; Sawilla 2009, S. 258. Zur protestantischen Auseinandersetzung mit Zeremonien nach Vorschriften der Interimstheologie vgl. ferner Bollbuck 2014, S. 49-50; Wengert 2006, S. 33-49; Moritz 2009, S. 234-251. In einem offenen Brief an Melanchton vom 20. Oktober 1549 meinte Flacius nämlich, dass die Adiaphora die Glaubenslehre wie auch den Ritus „papistisch“ umgestalten würden. Vgl. hierzu Falcius, Responsio. 
auf, die eine Kontinuität zwischen den Apostolischen Konstitutionen und der Tridentiner Kirche juristisch auslegen.

Um eine solche Fortführung darstellen zu können, betrachtete Francisco Torres die pseudoisidorischen Dekretalen als eigentliche Rechtsgrundlage für die drei zentralen Bereiche von doctrina, caeremonia und ecclesiastica politica. Damit ist eine Verbindung zwischen den Correctores Romani und einem genuin kurialen Verständnis von Kirchengeschichtsschreibung zu erkennen. Die Adversus Magdeburgenses Centuriatores des Jesuitentheologen Torres sind somit eher als ein Produkt dieser Gruppe von Rechtsgelehrten zu betrachten als ein kirchenhistoriographisches Werk der 1569 einberufenen Congregatio antimagdeburgica. Marry E. Sommar gelang es anhand der in der Biblioteca Vaticana aufbewahrten Handschriften der Correctores Romani nachzuweisen, dass sich diese Gruppe kurialer Rechtsgelehrter bei der Entstehung des neuen und erst 1582 gedruckten Decretum Gratiani ausführlich mit den pseudoisidorischen Dekretalen auseinandersetzte. ${ }^{489}$ Wie dieser römischen Kirchenrechtssammlung zu entnehmen ist, bestand die Arbeit der Correctores - und somit auch des spanischen Jesuitentheologen Francisco Torres - darin, die Autorschaft der in der Causa II enthaltenen Dekrete mittels einer textkritischen Untersuchung Päpsten aus der Spätantike zuzuschreiben. Einen wichtigen Beitrag dazu leisteten die Arbeiten Francisco Torres' zu den pseudoisidorischen Dekretalen, denen sich der Jesuitentheologe seit 1568 annahm und dies als zweites sowie drittes Buch in seine Adversus Magdeburgenses Centuriatores aufnahm. ${ }^{490}$ Darin findet sich ein zentraler Abschnitt über das Zeremoniell der Weihe eines neuen Kirchenbaus, der in dem später erschienenen Caeremoniale Episcoporum ebenfalls auftaucht.

Der Abschnitt De consecratione ecclesiarum beginnt mit der einleitenden Bemerkung, dass die Zenturiatoren die Herkunft des traditionellen römischen Messritus aus der apostolischen Tradition nicht anerkennen würden. Sowohl Papst Clemens I. als auch Felix IV. hätten aber in ihren Briefen ausdrücklich auf diese apostolische Tradition, die allen Bischöfen die Weihe von Kirchen samt der damit verbundenen Messfeier zusprach, hingewiesen. ${ }^{491}$ Indem sich Torres hier ausdrücklich auf die von Papst

489 Sommar 2002, S. 59 für eine Analyse der Handschrift BAV, Vat. lat. 4913, f. 82r-106r.

490 Pogiani, Epistulae, III, S. 299: „Torrensis ingressus est collegium Jesu Romae et se commendat D. V. illustrissimae quamplurimum; die epiphaniae se obtulit domino cum tribus Magis in holocaustum. Agressus est duos tractatus contra centuriatores alterum, quod epistolae Clementis, Anacleti, Fabiani cum citant apostolorum traditionem, non mentiantur: alterum, quod earum epistolarum doctrina sit scripturis sanctis conformis. Et hoc tractatus citius in luces praestabit“. Vgl. auch das Briefschreiben Borjas‘ an Canisius zwischen 1568 und 1569 in Braunsberger, Canisius Epistulae et acta, VI, S. 169, 186. Die Antwortbriefe Canisius' an den Jesuitengeneral finden sich in ibid., VI, S. 334-345, 367.

491 Torres, Adversus Magdeburgenses Centuriatores, S. 217: „[...] quam ille ad omnes Episcopos de consecratione ecclesiarum ad celebrandum Missas scripsit, sic ait: Quamobrem fratres hortamur vos, monemus, \& flagitamus, ut à tramite Apostolicae institutionis nequa quam re cedatis, nec à capite dissideatis: sed fidem \& ordinem, quem Apostoli \& Apostolici viri statuerunt [...]“. 
Felix IV. verfasste De consecratione ecclesiarum bezieht, verbindet er diesen zeremoniellen Aspekt mit der entsprechenden Stelle im Decretum Gratiani, die den Correctores Romani unter der Maxime Necessitas non habet legem zumindest bekannt gewesen sein musste. ${ }^{492}$ In dieser Gesetzgebung ist auch die Erlaubnis enthalten, die Messe auch ausserhalb geweihter Kirchen feiern zu dürfen, wenn dies für nötig gehalten wird. Im Verständnis der Kanonistik wird der Kirchenbau durch die rituell ausgeführte consecratio zum eigentlichen Raum, in welchem die Gnade Gottes gespendet wird, erhoben. Torres bedient sich dabei insbesondere der vierten Predigt des Ivo von Chartres (De sacramentis dedicationis) und erläutert damit den sakramentalen Charakter des Einweihungszeremoniells. ${ }^{493}$ Francisco Torres verwendet die Kirchenweihe nicht nur dafür, um die sakramentale Dimension des Zeremoniells an sich zu betonen. Im Zeremoniell der Kirchenweihe wird nämlich insbesondere die Sakramentalität des zelebrierenden Bischofs - in diesem Falle des Papstes selbst - betont. ${ }^{494}$

Mit seinen Adversus Magdeburgenses Centuriatores hat der spanische Jesuitentheologe und Mitarbeiter der Correctores Romani Francisco Torres einen entscheidenden Beitrag zur Widerlegung der Zenturien geleistet, indem er die Kirchengeschichtsschreibung als Artikulation der zeremoniell-sakramentalen Einsetzung nicht nur des Bischofsamtes, sondern auch des Papstamtes anhand der in den pseudoisidorischen Dekretalen beschriebenen Riten auffasste. Die Zenturiatoren hatten bereits im zweiten Band ihrer Ecclesiastica Historica festgehalten, dass die in diesen Dekretalen überlieferten Bestimmungen, die sich in den Briefen der Päpste Anaclet, Alexander, Hyginus und Eleutherius wiederfinden, eigentlich den Aposteln zuzuschreiben sind. Da die Apostel aber solche Schriftstücke selber nie verfasst hatten, mussten alle diese Bestimmungen aus späteren Zeiten stammen. ${ }^{495}$ Gleichzeitig nahmen die Zenturiatoren damit

492 Corp. iur. canon., ed. Rom. (1582), De consecratione, D.1.c.11, resp. C.1.q.1. Vgl. auch für die Epistola ad omnes episcopos Papst Felix' IV. insbes. PL, Bd. 65, Sp. 15-23.

493 Torres, Adversus Magdeburgenses Centuriatores, S. 225. Für die Predigt Ivo v. Chartres‘ vgl. PL, Bd. 162, Sp. 527-535. Zur Bedeutung Ivo v. Chartres für die Kanonistik vgl. dann Rolker 2010, S. 149; Fassler 2010, S. 133-136; Brasington 2006, S. 129-147.

494 Torres, Adversus Magdeburgenses Centuriatores, S. 225: „Pontificum ad ostium accedere basilicae clero \& populo susequente \& percutere liminare cum virga, \& cantare antiphonam „Tollite portas \& c.” \& haec quidem, inquit, nequaquam, ut pueris aut pureliter intelligentibus videtur, quasi ludus accipienda sunt“.

495 Ecc. Hist., II, 147: „Nec ordinationis ritus, qui Anacleti epistula 2 describitur de impnenda 4. Evangeliis et oleo chrismatis hoc seculo in usu fuit. Nec tempori et veritati congruum illa decreta de appellatione ad Romanam sedem, de comparitione, de Episcopo pulso ante iudicium restituendo, de sacris vasis non tangendis nisi ab hominibus sacratis neque a monachis aut sacris foeminis, de aqua et sale benedicenda et aspergendo populo ut sanctificetur, de non servandis chirographis in extortis: qualis passim extat in Anacleti, Alexandri, Hygini, Eleutherii litteris. Nam ab apostolis, quibus pleraque adscribuntur, nusquam statuta leguntur et a doctrina eorum prorsus abhorrent“. Zum Umgang mit den pseudoisidorischen Dekretalen in den Magdeburger Zenturien vgl. insbes. Hartmann 2002, S. 205-206; Fuhrmann 1972-1974, Bd. 1, S. 5-6. Bereits Flacius hatte in seinem Catalogus testium veritatis eine Grundlage für den Fälschungsnachweis der Dekretalen mittels sei- 
die früheren Päpste in Schutz, da sie diese nicht als Verfasser solcher Artikel betrachteten. Für die Zenturiatoren ist daher der Kirchenhistoriker Eusebius der vornehmste Zeuge für den Nachweis des Fälschungscharakters dieser Dekretalen. Obwohl er eine bibliotheca instructissima besass und mit Kaiser Konstantin d. Gr. eng befreundet gewesen war, hatte er doch nichts von diesen Papstbriefen zu berichten gewusst. ${ }^{496}$ Francisco Torres musste sich mit seiner Bemerkung, die Zenturiatoren hätten selbst durch ihren Materialreichtum das geeignete Rüstzeug zu ihrer Widerlegung bereits vorgelegt, auf die in Schutz genommenen Päpste aus dem Urchristentum bezogen haben. Die Nadel im kirchenhistoriographischen Kompass versuchte er durch das Tripel von doctrina, caeremoniae und ecclesiastica politica nun auf die Kontinuität der Apostolischen Konstitutionen in diesen Dekretalen auszurichten. Dafür dienten ihm die caeremoniae als zentrale Grundlage, um die Aufrechterhaltung einer apostolischen Sakramentalität im Ritus der Bischofsweihe nachweisen zu können.

Francisco Torres gab mit seinen Adversus Magdeburgenses Centuriatores auch den Takt für die Widerlegungsarbeiten der Congregatio antimagdeburgica vor. Eine gezielte Widerlegung der Magdeburger Zenturien sollte nämlich nicht die Altertümlichkeit kirchenhistoriographischer Entwicklungen der Romana Ecclesia nachweisen, wie dies noch der Augustinermönch Onofrio Panvinio beabsichtigt hatte, sondern vor allem die Kontinuität zwischen den Apostolischen Konstitutionen und der gegenwärtigen römischen Kirche zeigen. Das Potenzial von Torres' Widerlegungsarbeiten stiess aber unter den Correctores Romani dennoch an seine Grenzen, denn der wichtigste Mitarbeiter dieser Kommission, Antonio Augustín, war mit diesem besonderen Umgang mit den pseudoisidorischen Dekretalen nicht einverstanden. Es war auch derselbe Augustín, der als erster die falsche Zuschreibung ihrer Autorschaft an Isidorus erkannte. ${ }^{497}$ Sein Dialogorum zeigt ferner, dass der Fälschungscharakter dieser Dekretalen in der Korrespondenz zwischen Augustín und einem weiteren Mitarbeiter der Correctores Romani, Miguel Thomás de Taxaquet,

ner Kritik der Pseudo-Clemens-Briefe geschaffen. Vgl. hierzu Flacius, Catalogus, S. 6, 57; Harder 2014, S. 173-186; Zechiel-Eckes 2001; Bollbuck 2014, S. 340-341. Es wäre noch eine eingehendere Untersuchung wert, inwieweit die pseudoisidorischen Dekretalen bereits im Carranza-Prozess womöglich Verwendung gefunden haben könnten, insbesondere mögliche Verweise auf die sogenannten Capitula Angilramni.

496 Ecc. Hist., II, 151: „Accedit insuper auctoritas aliorum antiquissimorum testium ad excusandos Romanos episcopos, ne harum epistolarum auctores credantur. [...] Nam Eusebius, qui Bibliothecam instructissimam habuit quemque cum Constantino imperatori Romano intimus esset, nihil potuisset latere memorabilium rerum Romanorum episcoporum, tantum Clementem et Victoris litterarum meminit“. Vgl. Hartmann 2002, S. 206. Dies trifft auch im Falle des Einweihungszeremoniells für Kirchen in Eusebius' Kirchengeschichte zu. Vgl. Eusebius, Historia Ecclesiastica, X.3, S. 412-413.

497 Augustín, Iuris Pontificii Veteris Epitome, II, n.p., Index eorum, quae in hac collectione posita sunt: „Epistulae Pontificum Romanorum [...] qui eidem Isidoro adscriberent, sed vehementer falluntur, nam ipse se scriptor prodit his carmininbus ineptis usus, quae detegunt eum adulescentem fuisse tum primum manum ferulae subtrahentem“. 
stark debattiert wurde. Die pseudoisidorischen Dekretalen müssen innerhalb dieser Gelehrtengruppe eine zentrale Rolle im Zusammenhang mit ihrem grossangelegten Editionsprojekt einer Neuausgabe des Corpus Iuris Canonici gespielt haben. ${ }^{498}$ In der Tat erwiesen sich die textkritischen Auseinandersetzungen mit den pseudoisidorischen Dekretalen des Jesuitentheologen Francisco Torres als zu schwach, um kirchenhistoriographisch die durch die Magdeburger Zenturien nachgewiesenen Fälschungen weiterhin genügend verteidigen zu können. Damit war nicht nur die Sichtweise der Zenturiatoren, sondern auch ihr kritisches Instrumentarium entschieden von den Instruktionen François Baudouins, in denen die pseudoisidorischen Dekretalen abgelehnt wurden, bestimmt. Die Zenturiatoren konnten in ihrer Arbeitsweise die rein polemische und konfessionell begründete Kritik von Matthias Flacius' Catalogus in eine allgemeine Kritik des Papsttums und der römischen Kirche umwandeln. 499

$\mathrm{Zu}$ der Einsicht, wie eine Kirchengeschichte überhaupt geschrieben werden musste, war die römische Kurie noch nicht gelangt. In einem Briefschreiben vom 9. Juli 1571 an seinen Ordensbruder Jeronimo Nadal erläutert Francisco Torres die Schwierigkeiten der Drucklegung seiner Adversus Magdeburgenses Centuriatores. Der spanische Jesuitentheologe überlegte sich sogar, seine Schrift nicht mehr in Florenz, sondern in Venedig drucken zu lassen, um nicht in das Visier der römischen Indexkongregation zu gelangen:

[...] y después caminando enla estampa cayo malo el compositor, y fue necesario esperar otro mes, y viendo que no convalecia, se tomo otro, que a la sazon se pfreció partirse de otra estampa, el cual al mejor tienpo tanbien falto por que se fue a Nevezia de manera que la enfermedad del compositor, y falta de otros que supliesen me ha hecho tardar tanto [...] tengo deseo de y ala obediencia de V. R. y de entender en meter en orden lo que tengo sobre la Scritura, por que esta es la voluntad de nuestro padre, para lo qual espero que V. R. me ayudara dandome ayuda, y lo primero sera sacra una cathena que tengo hartos annos ha sobre los 12 . prophetas menores sacada delos interpretes griegos Cyrill, Theodoro Antiocheno, Theodorato, y añadire Didymo y delos latinos Hieronumo [...]..$^{500}$

Die Ansicht der bisherigen Forschung, die Adversus Magdeburgenses Centuriatores könnten als Produkt einer als „Literatur-Kongregation“ einberufenen Kardinalskommission betrachtet werden, muss den hier dargelegten Ausführungen zufolge

498 Augustín, Dialogorum, S. 55-70. Zu Taxaquets Beteiligung an den Revisionsarbeiten zum neuen Decretum Gratiani vgl. zuletzt Sommar 2002, ab indicem.

499 Lyon 2003, S. 266-268; Bollbuck 2014, S. 341; Erbe 1978a, S. 82. Vgl. auch Baudouins Verhältnis zu den liturgischen Arbeiten Georg Cassanders in Erbe 1978b, S. 537-560. Cassander hatte zusammen mit Cornelius Wouter in Köln den Zenturiatoren Handschriften Ivo von Chartres zur Verfügung gestellt, die in Siegburg gefunden wurden. Vgl. hierzu insbes. Nolte 1951, S. 52-54; Strüwe 1980, S. 57, 61-62.

500 ARSI, Ital. 142: Epistolae Italiae 1571, f. 100r. Am 3. Februar 1572 bat Torres Nadal um ein privilegio o motu proprio für die Drucklegung seiner Abhandlung: ivi, Ital. 143: Epistolae 1572-1573, f. 112r. 
neu bewertet werden. ${ }^{501}$ Aller Wahrscheinlichkeit nach wäre Matthias Flacius` Catalogus von der 1571 einberufenen Indexkongregation ohne grössere Schwierigkeiten widerlegt worden, denn zu diesem Zeitpunkt verfügte die Kurie über genügend polemische Widerlegungsarbeiten. Aber das Werk der Magdeburger Zenturiatoren stellte keineswegs eine polemisch verfasste Kirchengeschichtsschreibung dar. Stattdessen wurde darin eine wesentliche Neubeurteilung der conditiones Ecclesiae und ihrer loci temporis vorgenommen, denen die Indexkongregation zum Zeitpunkt ihrer offiziellen Einberufung bei Weitem noch nicht gewachsen war. ${ }^{502}$ Die Widerlegungsversuche Konrad Brauns, Wilhelm Eisengreins, Onofrio Panvinios und des Jesuiten Petrus Canisius hatten den aus dem Blickwinkel der Correctores Romani und des Jesuitentheologen Francisco Torres wichtigen Einbezug der kirchlichen Jurisprudenz und ihrer Dekretaliensammlung in eine Darstellung der Kirchenentwicklung weitestgehend nicht beachtet, weshalb diese ersten Ansätze bis 1571 auch zwangsläufig scheitern mussten. Sie können sogar als zum Scheitern verurteilte Arbeiten bezeichnet werden.

Der Jurist François Baudouin hatte in seiner Institutio historiae universae von 1561 auf der Grundlage seiner umfassenden Erforschung der Geschichte menschlicher Gesellschaft als Ausdruck ihrer Rechtsverhältnisse das göttliche Prinzip in ein dreiteiliges Schema von historia rerum humanarum, historia naturalis und historia divina eingeteilt. Damit schuf er einen geeigneten Ausgangspunkt, wovon ausgehend der Begriff der historia universa verstanden werden konnte. Integrale Bestandteile einer solchen Geschichtsbetrachtung bildeten Geographie, Chronologie und die Kenntnis der biblischen Bücher, aber auch die Berücksichtigung unterschiedlicher Quellensorten, die literarische und schriftliche Überlieferungen sowie Münzen, Inschriften und Kunstwerke umfassten. Baudouin zufolge sollten diese einer rigorosen Quellen- und Echtheitskritik unterzogen werden. ${ }^{503}$ Auch wenn sich dieses Kriterium in den Adversus Magdeburgenses Centuriatores des Jesuitentheologen Francisco Torres nicht finden lässt, hatte der Jesuitentheologe nichtsdestotrotz diese Idee der historia tripartita für die Arbeiten der römischen Kurie - insbesondere für die 1572 neu einberufene Congregatio Ger-

501 Zuletzt dazu Benz 2002, S. 35, der anhand Orella y Unzue 1976, S. 170-175 eine falsche Beurteilung dieser Kongregation vornimmt.

502 Vgl. den Brief Baudouins an die Zenturiatoren vom 13. Juni 1556, abgedruckt in Erbe 1978a, S. 266: „Nam id primum esse necesse intelligebam, ut quo iure quave conditione Ecclesia eius loci temporisque fuisset, recte intelligerem. Tum vero, ut ipsius Ecclesiae statum proprius cognoscerem: quae religionis doctrina, quae caeremoniarum forma fuisset, considerabam, ut et in illius tamquam animam, et in corpus faciemque intuerer“.

503 Kelley 1964, S. 35-57; Franklin 1963, S. 116-136; Erbe 1978a, S. 110-114, 217; Bollbuck 2014, S. 334-335. Ein solches Geschichtsverständnis stützte sich insbesondere auf Ciceros leges historiae. Vgl. hierzu dann Baudouin, De Institutione historiae universa, S. 640: „Quae quidem omnia non modo alijs, sed sibi ipsis magis prescripserunt, que observarent. Testem ex multis unum cito Polybium. Neque si hoc illi non fecissent, aliunde potius quam ab Cicerone tales leges repetendas esse putarem qui quod de officio historicorum dici posset, optime \& brevissime exposiot libri II. de Oratore“. 
manica - verwenden können. Torres betrachtete die caeremoniae und die damit verbundenen Riten als einen Bestandteil der Grundlagen der Glaubenslehre und der Kirchenverwaltung, welche zusammen die conditione Ecclesiae ausmachten. Eine juristische Auseinandersetzung mit der Historia Ecclesiastica sollte daher zwangsläufig auf der Grundlage des kanonischen Rechtes sowie der sichtbaren Offenbarung von Zeremonien und Riten stattfinden. Hat sich diese entscheidende Neuausrichtung auch auf die Arbeitsweise der Congregatio Germanica im Boncompagni-Pontifikat Gregors XIII. ausgewirkt?

\subsection{Die Arbeitsweise der Congregatio Germanica}

Diese Frage, welche unweigerlich auch eine Neubeurteilung der Arbeitsweise dieser Kongregation fordert, muss noch einmal auf der Grundlage des bereits geschilderten Problems, wie die kirchenhistoriographischen Arbeiten gegen die Magdeburger Zenturien von Spanien nach Rom überführt wurden, beantwortet werden. Die Briefkorrespondenz zwischen dem Kardinal Galli und dem spanischen Nuntius Monsignore Juan Bautista Castagna bietet eine geeignete Ausgangslage, um den Zusammenhang der ab 1572 einberufenen Kardinalskongregation und der Indexkongregation neu zu beurteilen. Die Frage, ob Spanien oder Rom als offizieller Arbeitsort gegen die Zenturien gelten sollte, war mit der Abreise Miguel de Médinas und Pedro Fuentidueñavon Rom nach Madrid verbunden. Hatte sich damit auch zwangsläufig der Arbeitsort verlegt und wie ist die durch Philip II. an der Universität von Alcalá einberufene Theologenkommission gegen die Zenturiatoren einzuordnen? In seinem Briefschreiben vom 29. August 1573 stellte der spanische Nuntius Castagna dem Kardinal Tolommeo Galli die Gründe vor, weshalb die Widerlegungsarbeiten gegen die Zenturien in Spanien und nicht in Rom stattfinden sollten:

\footnotetext{
Parlai delle Centurie et Sua Signoria approva molto, che vi si risponda, facendosi un' historia Ecclesiastica per la quale si dimostri la verità delle cose catoliche et si scopra et dimostri la falsità del historia heretica. Et concludemmo, che è meglio farla qui, che in Roma, et si risolse, chel Fontidomo fosse uno de principali, che vi havesse d'attendere et per la dottrina et per la lengua, dandoli altri homini in aiuto per far presto, Et all‘ ultimo restammo, che alla venuta di Sua Maestà, s‘habbi da stabilir il negotio circa il quale si darà poi aviso di quello che sarà concluso..$^{504}$
}

Es sei bereits vorweggenommen, dass Galli in seinem Antwortschreiben an Castagna diesen Vorschlang nicht befürwortete. Stattdessen erklärte der Kardinal lediglich, dass die Widerlegung der Zenturien dem Papst similmente caro seien. Dies bedeutet aber nicht, dass Gregor XIII. an Spanien als neuen Arbeitsort dachte, wie dies Benz jüngst annahm. ${ }^{505}$ Das Briefschreiben aus Madrid an Galli sagt nämlich nur, dass man zum Entschluss gekommen sei, die Arbeiten zu verlegen. Von der Errichtung

504 ASV, Segr. Stato, Spagna, 7, f. 417r; Baumgarten, Hispanica IV, S. 40-41.

505 ASV, Segr. Stato, Spagna, 15A, f. 246v; Benz 2002, S. 36. 
eines eigenständigen Arbeitskreises in Spanien ist nicht die Rede. In der Tat wurde die bereits seit langer Zeit anstehende Frage nach einer risposta alle Centurie nicht einmal im November desselben Jahres gelöst. Der Nuntius Castagna schlug dann weitere Personen vor, die sich neben Fuentidueñas einer solchen Arbeit ebenfalls widmen könnten. In diesem Zusammenhang erwähnt er auch Francisco Torres. ${ }^{506}$

Dass Rom weiterhin als Arbeitsort und damit die neu einberufene Congregatio Germanica bestehen sollten, geht aus einem an Kardinal Giovanni Commendone adressierten Briefschreiben vom 11. November 1573 hervor:

Questi Padri del Giesù, et in spetie il Padre Canisio mi dicono, che come da molte persone pie, et intelligenti sparse per Germania vien questo studio charitatevole, che si mette da N. Signore in procurar' sanità alle profunde, et quasi infistolite piaghe del paese sommamente commendato, elevandossi già gl'anomi à qualche speranza d'importante profitto [...] che nel medesimo tempo [...] si premesse d'ordine di S. Beatitudine in proseguir' la risposta alle Centurie Magdeburgensi, come quelle, che per la qualità delle materie loro sono avidamente lette, et per il veleno, che contengono studiando di abrogar' l'authorità alla Chiesa Santa et alle traditioni di essa, sono per apportar' se non saranno confutate troppo notabil danno, et perche sarà questa occupatione lunghissima [...] io ho commendato il ricordo [...] che si vadi pensando da pij, et dotti: ma sapendo, che non si truovano così facilmente huomini per queste imprese, sono andato sottranendo con destra maniera, se si potesse haver' qualche parte d'ajuto da chi s'è havuto l'avertimento [...] che N. Signore non mancherebbe di promuover' essendo bisogno, con la liberalità suo sì buona opera [...] allegandossi il poco numero d'huomini della provintia, con l'occupationi, c'hanno quelli, che sarebbono à proposito della Compagnia, di governi di collegij, di lettioni pubbliche, d'administrationi de‘ Sacramenti, per le quali è impossibile, che attendino ad impresa simile, che ricerca tutto l'uomo et non una, ma molti. ${ }^{507}$

506 ASV, Segr. Stato, Spagna, 7, f. 517r; Baumgarten, Hispanica IV, S. 41-42: „Non si manca di sollecitar quanto si puo, che si venga à qualche bona rissolutione di dar principio alla risposta alle Centurie. Et parlando io doi di sono con il Signor Presidente del Conseglio Regio, si fece qualche discorso sopra questa materia. Il detto Signore è di parere, che sia bene, che la risposta si faccia qui piu presto che in Roma per alcune bone ragioni, che altre volte sono state havute in consideratione. Il modo che s'hà da tenere nel confutar le Centurie è che senza far mentione di questi libri, si componga à parte uni historia Ecclesiastica, nella quale si va demonstrando la verità delle cose successe nella Chiesa Catholica di tempo in tempo, dalla qual verità si venga à far manifesta la falsità del historia de gli heretici confirmando etiam con ragioni, ove sarà bisogno, la parte di Catolici, col rispondere à quelle de gli heretici. Gli homini à quali principalmente s'hà da commetter questa nobile impresa, pare al detto Signor Presidente, che possino essere à proposito il Fontisdonio, che hà cominciato à lavorare in questo, Arias Montano, che hà faticato tanto in quella gran bibbia di tante lingue et hà fatto tante altre degne cose, il quale hora stà in Fiandra, il Dottor Torres Jesuita, che è in Roma, et il Dottor Villapando, Cadredatico in Salamanca. Gli piace anche, che Monsignor (Diego) di Lerida ne sia partecipe, cioè che di quello, che questi andaranno tessendo alla giornata, si dia conto al detto Monsignore, et ancora ad alcuni qui. Il luoco, dove questi quattro hanno da star ritirati da ogni altro negotio, sarà Alcala, universita principale in questo Regno et qui vicina, ovè sarà commodità de libri et de molti, che possono aiutare“. Vgl. ASV, Segr. Stato, Spagna, 15A, f. 271v, Antwortschreiben Gallis.

507 Braunsberger, Canisius Epistulae et acta, VII, S. 683-684; NBD, III, S. 217, wo Torres, Sirleto, Hosius und Paleotti als mögliche Stützen innerhalb der Kongregation in Frage kämen. Hierzu dann 
Während ihrer ersten Sitzung am 2. Dezember 1573 betrachteten die Kongregationsmitglieder ebenfalls Rom statt Spanien als bevorzugten Arbeitsort. ${ }^{508}$ Der vom spanischen Nuntius Castagna vorgeschlagene Arbeitskreis an der Universität von Alcalá sollte demgemäss nur Privatarbeiten leisten, denen sich die neue Congregatio Germanica durchaus bedienen sollte. Diese von Philipp II. in Spanien einberufene und sich vornehmlich aus Theologen zusammensetzende Kommission kann aus einem Brief ihrer beiden wichtigsten Mitglieder, Miguel de Médina und Pedro Fuentidueña, an den Nuntius Castagna rekonstruiert werden. Sowohl Fuentidueña als auch Médina waren der Meinung, dass eine gezielte Widerlegung der Zenturien auf der Grundlage einer Tridentiner Theologie des sichtbaren Priestertums und des Messopfers Christi stattfinden müsse. Fuentidueña legte diese Strategie bereits in seinem Traktat $\mathrm{De} s \mathrm{a}$ cerdotio et sacrificio christiano contra Centuriadores vor. ${ }^{509}$ Zudem waren die beiden spanischen Franziskanertheologen der Meinung, dass die Universitätsbibliothek von Alcalá bessere Bedingung als die in Rom biete. Der reiche Bestand der Universitätsbibliothek würde es der Theologenkommission ermöglichen, den Apostolischen Stuhl noch stärker gegenüber der protestantischen Kritik verteidigen zu können. ${ }^{510}$ Die beiden Theologen Médina und Fuentidueña waren sich durchaus bewusst, dass die Magdeburger Zenturien das Produkt einer kollektiven Zusammenarbeit waren und deren Widerlegung erst mit einer ähnlichen Idee einer Schreiberstube zum Erfolg füh-

auch Klaiber 1978, S. 210-213; Orella y Unzue 1976, S. 407-410; Benz 2003, S. 36 hinsichtlich einer ersten Folge dieser Entscheidung, den Franziskaner Johannes Nas zu unterstützen.

508 Schwarz, Briefe und Akten, II, S. 82.

509 ASV, Segr. Stato, Spanga, 17, f. 45r: „[...] atque ita multo consultis esse duxit, id fieri extra Urbem congregationemque dissoluit et fratri Michaeli Medinae, qui eius iussu de penitentia magnum iam volumen confecerat, atque doctori Petro Fontidonio, cui fuerat ab eodem iniunctum argumentum de sacerdotio et sacrificio Christiano, licentiam veniendi in Hispaniam concessit precepitque, ut in eadem provincia scribendi contra centurias perseveraremur et nobis per breve apostolicum dedit facultatem [...]“. Der Traktat lässt sich somit auf das Jahr 1571 datieren, da Fuentidueña offensichtlich dieses aus seinen Predigten für Pius V. zwischen 1570 und 1571 in Rom zusammenstellte. Vgl. Fuentidueña, Oratio habita ad Pium V, in idem., Apologia pro sacro \& oecumenico Concilio Tridentino, S. 306-373. Zur Entstehungsgeschichte der Theologenkommission in Alcalá vgl. insbes. Orella y Unzue 1976, S. 347-360.

510 ASV, Segr. Stato, Spanga, 17, f. 45r: „Quod ad hoc negotium perficiendum extra Urbem, nullibi commodius et brevis confici posse, multi arbitrabantur, quam in Hispania, cum ob Catholici Regis studium in Christianam Religionem, qui libentissime susciperet hoc munus tam pium et tam necessarium, facile suppeditaret, tum etiam ob ingentem et locupletissimam bibliothecam, quam habet refertissimam omni librorum genere collectam et multis bibliothecis doctissimorum hominum et ex varijs provincijs, unde sibi libros adducendos curavit, nec desunt in hispania homines docti linguarum et antiquitatis periti, qui id munus possent explere, atque ea ratione illa omnia, quae ex provincia atque regno per dei gratia adeo catholicis prodirent, minus essent hereticis suspecta, minoremque illis occasionem calumniandi praeberent, maiusque pondus haberent ad propugnandam apostolicae sedis autoritatem, quam ex professo centuriatores isti expugnare conantur“. Dieselbe Argumentation findet sich ebenfalls in einem memoriale Fra Luigis in BAV, Reg. lat. 2020, f. $217 r$. 
ren konnte. Währenddessen begann die römische Indexkongregation unter der Leitung Guglielmo Sirletos bereits, sich zu einer solchen Arbeitsgruppe zu organisieren.

Die neue Indexkongregation kann als eigentliche Programmstifterin gelten, welche die Arbeitsweise der römischen Congregatio Germanica gegen die Magdeburger Zenturien leitete. Die Sitzungen der Congregatio Germanica und deren Protokolle, die im Borghese-Archiv überliefert sind, wurden zwischen 1573 und 1578 als Grundlage für Entscheidungsprozesse der Nuntien in Deutschland genutzt. Der bereits erwähnte Discorso Zaccharia Delfinos hatte die Instruktionen an die Nuntien in Deutschland zur obersten Aufgabe der Cogregatio Germanica bezeichnet. Um die Anbindung Deutschlands an die Kurie wiederherstellen zu können, mussten die Nuntiaturen notwendigerweise ausgebaut werden. ${ }^{511}$ Mit der Einsetzung dieser ersten „Reformnuntiaturen“ keimte die Hoffnung einer Rückführung der Protestanten in den Schoss der römischen Kirche von neuem auf. Die Congregatio Germanica wurde bislang nur hinsichtlich dieser „Reformnuntiaturen“ untersucht. Sowohl ihre Arbeitsweise in Bezug auf die Frage, wie eine Widerlegung der Magdeburger Zenturien stattfinden sollte, als auch ihre Zusammenarbeit mit der Indexkongregation wurden dabei vernachlässigt.

Die enge Zusammenarbeit zweier Kongregationen innerhalb der Kurie hatte auch die Einsetzung einer Theologendeputation in der Congregatio Germanica zur Folge. Diese Theologen mussten eine entsprechende Widerlegung der libri haereticorum vorlegen. ${ }^{512}$ Das Protokoll zur Sitzung vom 2. Dezember 1573 gibt preis, dass der bereits erwähnte Ratschlag Portias an den Kardinal von Como über die Einsetzung des Jesuitenordens gegen die Zenturiatoren umgesetzt wurde. In diesem Brief wird Kardinal Guglielmo Sirleto als Verantwortlicher dieser Strategie genannt. Ihm wurde die Aufgabe anvertraut, Materialien für eine Historia Ecclesiastica zusammenzustellen. ${ }^{513}$ Ein am 19. Juli 1574 verfasster Brief des Jesuiten Petrus Canisius an

511 Krasenbrink 1972, S. 95-192. Die Protokolle der Kongregation finden sich in ASV, Fondo Borghese I, 221 und liegen in Schwarz, Briefe und Akten, II, S. 38-41, transkribiert vor. Der Ausbau der Nuntiaturen wurde ebenfalls von Canisius befürwortet: „Nos magni faciendum putamus hoc medium atque remedium, nec ullum apparet efficacius, ad Religionem catholicam in Germania tum conservandam, tum restituendam“. Zit. n. Braunsberger, Canisius Epistulae et acta, VI, S. 286.

512 Dies geht aus dem Protokoll für die Sitzung vom 20. Juni 1573 hervor: „De deputandis theologis, qui respondeant libris haereticorum. Si agendum sit de rebus gravioribus, conquiratur ex omnibus partibus doctissimi theologi, de quorum consilio fiat Romae responsum summa maturitate. De libellis vero minoris momenti, respondendum esse in Germania et dandum negotium Jesuitis, qui vel respondeant ipsi, vel qui apti sunt ad respondendum indicent“. Zit. n. Schwarz, Briefe und Akten, II, S. 77.

513 Schwarz, Briefe und Akten, II, S. 82: „De Centuriis Magdeburgensis. Respondendum eis omnino esse. Et quamvis negotium hoc coeptum sit in Hispania et ibi absolvi possit, quam ad rem iuvandam mitti illuc possent aliqui homines docti, tamen hoc idem fieri debere in urbe, praesertim quod attinet ad compilationem ecclesiasticae historiae, qua in re cardinalis Sirletus plurimum conferre poterit“. Diese Theologendeputation löste daher eindeutig auch den in Alcalá eingesetzten Arbeitskreis um Médina und Fuentidueñaab. 
den Ordensgeneral Mercuriano erlaubt es, die Jesuiten zu identifizieren, die an einer solchen Arbeit ebenfalls beteiligt waren: Diego de Ledesma sollte sich einer Widerlegung der Abhandlung über die Indulgenzen in Chemnitz' Examen decretorum concilii Tridentini widmen, während Francisco Torres eine Verteidigung der Theologie des Purgatoriums übernahm. ${ }^{514}$ Den Ablässen und dem Purgatorium hatten sich bereits dreissig Konzilstheologen während des in Bologna tagenden Konzils gewidmet. Diese Theologen bestätigten die Existenz eines Reinigungsortes, der zwischen Himmel und Hölle angesiedelt war. ${ }^{515}$ Das am 3. Dezember 1563 erlassene Dekret bestätigte diese Tridentiner Glaubenslehre und wies die Aufsicht über deren Inhalte den Bischöfen zu. Sowohl die angemessene Verwaltung der Indulgenzen als auch der Glaube an das Purgatorium fielen damit in die pastorale Zuständigkeit der Bischöfe und nicht in diejenige der Kurie. ${ }^{516}$

Die Konzilsbeschlüsse hatten aber keine theologische Erklärung für Ablässe und für das Fegefeuer geliefert. Dementsprechend musste im neuen Catechismus Romanus von 1566 der Glaubensartikel zum Fegefeuer neu aufgegriffen und kontroverstheologisch begründet werden. Der Catechismus begriff das Fegefeuer als einen Ort, an dem die Seelen der Frommen eine bestimmte Zeit lang gepeinigt und geläutert würden, um ihnen Eintritt in die aeterna patria gewährleisten zu können. ${ }^{517}$ Der Glaubensartikel im römischen Katechismus bezieht sich nicht nur auf die Existenz des Fegefeuers, sondern behandelt auch die Möglichkeit und Wirksamkeit der Fürbitten der Gläubigen für die Seelen im Purgatorium. Die Gläubigen werden nämlich aufgefordert, Messopfer, Gebet und Almosen zum Nutzen der Seelen weiter zu pflegen. Insbesondere das Messopfer sollte „nach sicherster apostolischer Überlieferung“ allen Gläubigen dargebracht werden. ${ }^{518}$

Dabei ist ein Wandel im Bewusstsein der kurialen Widerlegungsarbeiten gegen die Magdeburger Zenturien festzustellen. Die beiden Jesuitentheologen Ledesma und Torres nahmen Melanchtons Theologie der loci communes auf und wandelten

514 Braunsberger, Canisius Epistulae et acta, VII, S. 228. Canisius war vor allem der Ansicht, dass eine Widerlegung der Zenturien unumgänglich auf einer Widerlegung des soeben fertiggestellten Examen Martin Chemnitz، aufbauen musste. Zur verzwickten Veranlassungs- und Entstehungsgeschichte dieses Werkes vgl. Mumm 1905, S. 1-34. Zum Examen dann Klug 1973, S. 1184-1187; Montgomery 1976, S. 73-94; Beutel 2007, S. 47-65.

515 CT, I, S. 666-675.

516 COD, S. 774; O’Malley 2013, S. 133, 243-244.

517 In diese aeterna patria „geht nichts Unreines ein”. CR, I, 6,3: „Praeterea est purgatorius ignis, quo priorum animae ad definitum tempus cruciatae expiantur, ut eis in aeternam patriam ingressus patere possit, in quam nihil coinquinatum ingreditur“.

$518 C R$, II, 4, 79. Dieser Wert der Messefeier kommt ferner in der Priesterweihe zur Sprache: Accipe potestatem offerendi sacrificium Deo, Missasque celebrandi tam ro vivis quam pro defunctis (CR, II, 7,25.) Zur protestantischen Abweisung des Fegefeuers vgl. Calvin, Institutio christianae religionis, III.5, S. 238-244. Die Zenturiatoren widmeten dem Fegefeuer ebenfalls einen kurzen Abschnitt: Ecc. Hist., XII, S. 759. 
diese in eine Grundlage des Heilsversprechens im Kreuzes- und Messopfer Christi um. Damit nahm sich die Kurie den loci communes Melanchtons und der Protestanten zum ersten Mal an und verlegte die Widerlegung der Zenturien in den Bereich der Kontroverstheologie. Ein wichtiger Beweggrund der Kontroverstheologie, sich der Kirchenhistoriographik anzunehmen, war, dass die polemischen Schriften der Franziskaner gegen die Zenturiatoren keine ausreichende Widerlegung dieser Kirchengeschichte präsentierten. Als Diego de Ledesmas De divinis scripturis in Köln erschienen, wurden die Grenzen solcher polemischer Widerlegungsschriften gegen die Zenturiatoren offenbart. Diese von Franziskanern geleiteten Arbeiten trugen zu einer schwierigen Zusammenarbeit mit den Jesuiten bei. Die Mitglieder der Congregatio Germanica wurden vom Nuntius Portia über das polemische Werk des Franziskaners Johannes Nas gegen die Zenturiatoren in Kenntnis gesetzt. ${ }^{519}$ In einem Briefschreiben vom 26. Januar 1574 an den Jesuitengeneral Mercuriano legte Petrus Canisius die Gründe sowie die Entwicklung des Misserfolgs dieser Franziskanerschriften dar. Die Jesuiten bezeichneten die Arbeiten des Franziskaners Nas als tragoedia. Canisius sieht die hauptsächliche Ursache des Scheiterns einer franziskanischen Widerlegung der Zenturien in der Ordenshaltung hinsichtlich der Frage nach der Häufigkeit des Kommunionsempfangs. In ihrem Inhalt unterschied sich die Überzeugung der Franziskaner deutlich von der Vorstellung einer libertas Christiana, welche die Jesuiten vertraten. Im Gegensatz zu den Franziskanern hatte der Jesuitenorden die Häufigkeit des Kommunionsempfangs mit dem Begriff der liberalitas communicandi umschrieben. Dieser hatte zur Folge, dass der Inhalt der Glaubenslehre des Kommunionsempfangs auf den eigentlichen Moment der göttlichen Sündenvergebung ausgerichtet wurde. Die Franziskaner hielten aber noch am Glauben fest, dass die Häufigkeit des Empfangs der Sakramente indirekt mit der Heilserlangung zusammenhänge. ${ }^{520}$ Dogmatisch betrachtet waren sich Franziskaner und Jesuiten in der Frage der Häufigkeit des Kommunionsempfangs uneinig. Für eine kontroverstheologisch orientierte Widerlegung der Magdeburger Zenturien musste daher zuerst die Frage geklärt werden, ob in der Häufigkeit oder im eigentlichen Moment des Kommunionsempfangs die Sündenvergebung stattfinde.

519 Schwarz, Briefe und Akten, II, S. 89: „De Patre Nassio. Laudandum de libro scripto et ut velit scribere alia contra mendacia adversariorum et scribat contra centurias, et si qua re opus erit [...]“. Zum päpstlichen breve für die Unterstützung durch Nas‘ Arbeiten vgl. NBD, III/3, S. 358-359 sowie ASV, Brevia Ap., Brevia Gregorii XIII, 28, ep. 76: „[...] vuluit ejus nomine his literis tuum hunc laborem a me commendari, ac simul significari, quando tua tali in re industria atque opera tanto ecclesiae Dei adjumento esse cernitur, cupere Sanctitatem Suam, ut Centuriis etiam Magdeburgensibus, quae (ut nostri) innumeris erroribus et mendaciis refertae sunt, respondendi consilium et laborem susciperes“.

520 Braunsberger, Canisius Epistulae et acta, VII, S. 172-176, bes. S. 173-174. Vgl. hierzu insbes. Dudon 1933, S. 274; Madrid, De frequenti usu sanctissimi Eucharistiae, S. 75. Zusammenfassend zur Jesuitentheologie des Kommunionsempfangs: O’Malley 1993, S. 152-157. 
Die Congregatio Germanica nahm sich einer kontroverstheologisch-dogmatischen Lösung dieses wichtigen Problems an. Damit berührte die Kongregation den sich im Boncompagni-Pontifikat manifestierenden Wandel des Kurienzeremoniells. Der englische Pilger Gregory Martin hielt in seinem Tagebuch die Bedeutung der Fronleichnamsprozession, mit welcher die Realpräsenz Christi im eucharistischen Leib der Hostie gefeiert wurde, fest. Während den zwischen 1576 und 1578 abgehaltenen Prozessionen zum Corpus Christi hatte Martin den täglichen Empfang der Kommunion beobachtet. ${ }^{521}$ Das Fronleichnamsfest markierte die Realpräsenz des vom Tod auferstandenen Christus und hielt sie in der geweihten Hostie fest. Während des Boncompagni-Pontifikates avancierte dieses Fest zum Vorbild zeremonieller Reliquienüberführungen, wodurch die Heiligkeit der körperlichen Überreste eines Heiligen entsprechend gewürdigt werden konnten. ${ }^{522}$ Der Zeremonienmeister Francesco Mucanzio hatte noch vor dem Jubeljahr 1575 die Sichtbarkeit dieser für das Kurienzeremoniell wichtigen Prozession in Bezug auf den sakramentalen Papstsegen erweitert. Sein Vorgänger Paris de Grassis vertrat noch die Auffassung, dass der Segen mit der Hostie unter pietatis praetextu gleichgesetzt war und der Segensakt per verbum oris et signum manus (,durch das Wort des Mundes und das Zeichen der Hand") vollzogen wurde. Mucanzio aber hielt in seinem Tagebucheintrag zu der am 21. Mai 1573 abgehaltenen Fronleichnamsfeier fest, dass der Segen bereits in Form des Kreuzzeichens verrichtet worden sei. ${ }^{523}$ Die in der Tradition des Kurienzeremoniells überlieferte Gleichrangigkeit zwischen gesprochener oratio und sichtbar vollzogenem signum wurde in der Performanz des Kreuzzeichens durch den päpstlichen Segen betont.

Im Kurienzeremoniell manifestierte sich sodann der Moment der Heilserlangung im Akt der Spende des Papstsegens am Schluss der Prozession. Die Kurie griff dieses Element im Zeremoniell zur Fronleichnamsfeier auf und wandelte es zu einer dogmatischen Widerlegung der Magdeburger Zenturien um. Die dogmatische Bedeutung des Papstsegens hatte Giorgio Vasari in seinem Fresko der Rückführung des Heiligen Stuhls von Avignon nach Rom durch Papst Gregor XI. in der Sala Regia bildsprachlich ausgelegt (Abb. 18). Diese Szene wurde bislang als „herrscherlicher adventus" und somit als triumphus interpretiert. Allerdings rückte Vasari mit seiner Bildlösung für diese Szene den Gegenstand der Rückführung des Heiligen Stuhls von Avignon nach Rom in die Nähe des kurienzeremoniellen Wandels der Fronleich-

521 Martin, Roma Sancta, S. 65-68, 89-91.

522 Vgl. infra, Kap. 8.2.

523 BAV, Chig. L.II.30, f. 124v: „Cantores dixernt versiculum Panem [...] et S.mus D. N. dixit orationem, et demum versa ad Populum facia vedit eum Sacramento benedictionem elevans illud ambabus manubus in forma Crucis. Quod tamen videtur factum fuisse praeter regulam quia cum Sacramento non debet fieri benedictio sed tamquam novus in officio non potui hoc previdere“. Vgl. auch zu de Grassis‘ Caeremoniale die Stelle in ASV, Fondo Borghese I, 568, f. 148r; Bölling 2004, S. 96-97. 
namsprozession unter Gregor XIII. Am 11. Dezember 1572 berichtete Vasari seinem engsten Korrespondenten in Florenz, Vincenzo Borghini, wie dieses Fresko entstanden war. Nach der Entscheidung Gregors XIII., den Hugenottenzyklus um eine dritte Szene zu erweitern und deshalb auf die Übergabe der Standarte an Don Juan d'Austria zu verzichten, wollte Vasari zwei Bilder malen, die den Namen des BoncompagniPapstes in „denkwürdiger Weise” feiern sollten:

Vorrei bene di questi Gregori pontefici trovar qualche cosa notabile, come quel Gregorio che ricondusse da Avignone la Sede Apostolica, e quello che levò l'autorità all'impero, che se la soscrizione suo non ci era, non era il papa bene eletto ecc., però aiuto che Sua Santità conosca che si va per via con qualche cosa che alluda d'ornamento a queste cose..$^{524}$

Besonders auffällig präsentiert Vasari die detaillierte Blickführung durch die Komposition der einzelnen Figurengruppen, die dem Betrachter die Dynamik des Prozessionszeremoniells im symbolischen Moment der Einnahme der Stadt durch Papst Gregor XI. vergegenwärtigt. Vasari lässt den Papst thronend auf seiner sedia gestatoria, die von den theologischen Tugenden Liebe, Glaube und Hoffnung getragen wird, auf dem Petersplatz einziehen. Gregor XI. blickt aus dem Bildfeld heraus und deutet mit seiner rechten Hand an, dass die Szene den Moment kurz vor seiner Spende des päpstlichen Segens darstellt. Vasari hatte sich dabei einer eigenen Vorlage bedient, die er in den Fresken der Sala dei Cento Giorni des Palazzo della Cancelleria für Papst Paul III. bereits verwendet hatte. Indem Vasari auf sein eigenes bildkompositorisches Repertoire zurückgriff, gelang es ihm seine malerischen Bildkompositionen schneller auszuführen. Die heilige Katharina von Siena weist dem päpstlichen Zug den Prozessionsweg, den die drei den Papst begleitenden Kardinäle auf ihren Pferden beschreiten sollen. Petrus und Paulus, die beiden Apostelfürsten Roms, schweben der Gruppe, die vom römischen Volk empfangen wird, voran. Eine in imperialem Harnisch und Helm gekleidete Figur wartet auf Gregor XI. und reicht ihm ein Zepter sowie eine Weltkugel. Zwei weibliche Personifikationen, welche die Zeichen sowohl der weltlichen (Zepter) als auch der spirituellen Macht (päpstliches Schirmchen) halten, warten auf den Papstzug. Ein Greis mit einer Amphore als Sinnbild des Tibers und der Wölfin mit den Zwillingen lagert zu ihren Füssen. Wie für die im Begriff des Segensaktes ausgeführte Papstfigur greift Vasari für diese allegorische Gruppe auf sein Bildrepertoire zurück. Dadurch verleiht er der Allegorie des imperialen Rom ihre würdige symbolische Wirkung. ${ }^{525}$

524 Frey 1923-1982, II, S. 731.

525 Die Vorzeichnung Vasaris aus dem Cabinet des Dessins zeigt die weiblichen Gestalten noch ohne ihre im Fresko dann hinzugekommenen Attribute, währenddessen Katherina von Siena ein Kruzifix in den Händen hält, auf welches in der Ausführung verzichtet wurde. Vgl. Taja, Descrizione, I, S. 27; Partridge/Starn 1990, S. 122; Egger 1948, S. 46; Kat. Paris 1972, S. 98-99; Kat. Ottawa 2009, S. 314-315. Böck 1997, S. 94-97, hielt an der Deutung dieser Szene in der adventus-Auslegung fest, obwohl sie anerkennt, dass Vasaris wohl bedeutendste Bildkomposition in diesem Zusammenhang sein Einzug Leos X. in Florenz im Quartiere di Leone X des Palazzo Vecchio ist. Dort setzte 
Im auffallend langen Bildtitulus wird angedeutet, dass diese Szene im Zusammenhang mit Vasaris Bedürfnis, diese noch vor der Fronleichnamsfeier im Jahr $1573 \mathrm{zu}$ vollenden, gedeutet werden kann: GREGORIUS XI PATRIA LEMOVICENSIS ADMIRABILI DOCTRINA HUMANITATEET INNOCENTIA UT ITALIAE SEDITIONIBUS LABORANTI MEDERETUR ET POPULOS AB ECCLESIA CREBRO DESILIENTES AD OBEDIENTIAM REVOCARET SEDEM PONTIFICIAM DIVINO NUMINE PERMOTUS AVENIONE ROMAM POST ANNOS LXXI TRANSTULIT SUI PONTIFICATUS ANNO SEPTIMO HUMANAE SALUTIS MCCCLXXVI. ${ }^{526}$ In Vasaris Fresko der Rückführung wird die Stadt Rom zum eigentlichen Ort der göttlichen Vorsehung und zum Sitz der Päpste erklärt. Darüber hinaus verweist die Bildunterschrift darauf, dass der päpstliche Sitz das Heilmittel gegen das kirchliche Schisma biete. Diese bewusste Betonung der Bedeutung Roms als Sitz des Heiligen Stuhls hatte ebenfalls Vasaris Korrespondent und engster Programmentwerfer für seine Fresken in der Sala Regia, Vincenzo Borghini, in einem auf den 17. Januar 1573 zu datierenden Briefschreiben folgendermassen festgehalten:

Quanto alle istorie: voi avete costì i maestri di queste inenzioni, che Roma n`è sempre stata fonte. Io posso così accennarvi qual cosa (più presto che trovare cosa nuova) sopra quelle che proponete, e delle fatte è superchio parlare. La quinta mi piace et è apropositissimo (quando sia così che delle istorie me ne riporto corta). [...] Per ornamenti ci suole esser certi rivesci di medaglie, che hanno ROMA RENASCENS o RESVRGENS, che farebbon a proposito, massimamento quando fussino accommodati al modo et uso cristiano più che al pagano, che non è difficile; $\mathrm{e}$ costì se ne sa quelche se ne può sapere: come dire mettergli in mano uno scettro di tre croci, esservi sopra la colomba dello spirito santo et simil cose, che accennino la religion cristiana. ${ }^{527}$

Borghini bezieht sich hier auf eine in den Pontifikaten Pauls IV. und Pius IV. verwendete Bildtradition der Roma resurgens, die hauptsächlich in Münzdarstellungen vorzufinden war. Auf diesen Münzen wurde die Allegorie der Stadt Rom als weibliche Figur in Harnisch mit einer bewussten Theologie des Heilsversprechens Christi bei seiner Wiederauferstehung verbunden. ${ }^{528}$ Vasaris Fresko der Rückführung verweist auf die Tradition der renovatio Romae. Allerdings wird sie nicht mehr länger als ein kontinuierlicher Prozess des päpstlichen Machtanspruchs dargestellt. Statt-

nämlich Vasari die prozessionell-triumphale Bewegungsdynamik des städtischen Eingeholtwerdens des Papstes mit der Blickführung gleich und zeigte den Medici-Papst, wie er auf seiner von einem Baldachin überwölbten sedia gestatoria auf die Piazza della Signoria getragen wird. Zur Instrumentalisierung des adventus für die päpstliche Bildprogrammatik vgl. insbes. Träger 1970, S. 71104, 80-84. Eine weitere Vorstudie zur Tiber-Gruppe am linken Ende des Bildfeldes befindet sich in den Uffizien (Inv.-Nr. 987S), welche Vasaris konzeptionelle Bildprogrammatik auf der Grundlage seiner Cancelleria-Fresken belegt.

526 Zur Enthüllung der Fresken Vasaris in der Sala Regia vgl. Frey 1923-1982, II, S. 785-786: „[...] la sala è finita et iermattina si scoperse con molta mia laude et onore [...]“".

527 Frey 1923-1982, II, 745.

528 Toderi/Vannel 2000, Bd. 3, Nr. 2237-2238; Corpus numismatum 2002-2015, Bd. 2, Nr. 476-478. Vgl. auch Röttgen 1976, S. 94-95, der in dieser Szene nicht nur die Erneuerung des Papsttums er- 
dessen vermittelt Vasaris Bildlösung einen bestimmten Moment innerhalb des christlichen Heilsgeschehens, den die Päpste mit ihrem Sitz in Rom einem jeden Gläubigen sichtbar vor Augen führen können. Die Stadt Rom wird somit als eigentlicher Ort des Heilsversprechens Christi im Kreuzes- und Messopfer betont. Dieser Ort des verwirklichten Heilsgeschehens erhielt im Kurienzeremoniell zur Feier des Corpus Domini seine deutlichste Ausprägung.

Die dogmatischen Widerlegungsarbeiten der neuen Congregatio Germanica weisen ein ähnliches theologisches Verständnis der Heilserlangung und der Sündenvergabe auf. Diese Arbeiten, die bis 1576 die Kardinalskongregation beschäftigten, betrachteten die protestantische Kirchenhistoriographie daher erstmals als theologisch-dogmatisches Werk, das die Mitglieder dieser Kongregation mit Hilfe einer angemessenen Auslegung der translatio imperii historiographisch zu widerlegen beabsichtigten. In diesem Zusammenhang war auch die Frage nach der Vorrangstellung Roms als eigentlicher Ort der Heilserlangung kirchenhistoriographisch zu beantworten, woraus sich dann eine dogmatische Grundlage des römischen Primats ableiten liess. Mit dem Phänomen der translatio imperii war ein „Übertragungsprozesses“ kaiserlicher Macht auf die Romana Ecclesia und auf das Papsttum angesprochen. Diese Machtüberführung wurde als theologischer locus behandelt, weshalb sie entsprechend der Stelle im Buch Daniel über den göttlich bestimmten „Wechsel der Zeiten und Fristen“ (Dan. 2,21) exegetisch gedeutet werden musste. Ihre kontroverstheologische Auslegung hatte direkte Folgen für ein kirchenhistoriographisches Verständnis der translatio imperii. ${ }^{529}$ Dogmatik und Kirchengeschichte begannen somit unmittelbar nach der Papstwahl Gregors XIII. sich gegenseitig zu beeinflussen. Diese bislang noch kaum wahrgenommene Wechselwirkung war jedoch nicht das Produkt der Congregatio Germanica. Die Bedeutung der translatio imperii wurde nämlich nicht, wie bislang angenommen wurde, von den Mitgliedern dieser Kongregation behandelt. Stattdessen war es die Indexkongregation unter der Leitung Guglielmo Sirletos, die sich einer Exegese der translatio imperii widmete. Mit ihren Zensuren zum kirchenhistoriographischen Werk von Carlo Sigonio aus Modena gelang es der Indexkongregation erstmals, die kirchenhistoriographische Widerlegung der Magdeburger Zenturien in die Bahnen der Bücherzensur umzuleiten. Bevor Cesare Baronios enge Zusammenarbeit sowohl mit der Congregatio Germanica als auch mit der Indexkongregation Sirletos untersucht werden kann, muss die Frage beantwortet werden, inwieweit die Zensureingriffe in Sigonios historiographische Arbeiten eine theologische Dimension aufgriffen, die mit

kennt, sondern auch richtigerweise feststellt, dass sie sich auf die Rückeroberungspolitik Gregors XIII. in den durch die Reformation verlorengegangenen Gebieten in Polen, Deutschland und Frankreich bezieht.

529 Zu dieser Exegese des Buches Daniel vgl. DThC 1924, IV/1, S. 55-75; Miegge 1995, S. 35-37. Bereits Luther hatte die translatio imperii als Fiktion abgetan: WA 54, S. 295-298. Zum Gesamtkomplex des protestantischen Umgangs mit dieser mittelalterlichen Theorie vgl. Goetz 1958, S. 281-304, wo auch Flacius‘ De translatione imperii Romani ad Germanos (1566) eingehend vorgestellt wird. 
der dogmatischen Grundsteinlegung der Kirchenhistoriographik seitens der Congregatio Germanica im Zusammenhang stand.

Carlo Sigonio widmete sich der Problematik der translatio imperii erstmals in seiner zwischen 1568 und 1571 verfassten Historia Bononiensis, die unmittelbar nach ihrer Drucklegung einer strengen Kritik seitens der römischen Zensurbehörde unterworfen wurde. McCuaig konnte anhand der in Argelatis Edition von Sigonios Opera omnia überlieferten Zensuren neben den Kardinälen Ugo Boncompagni und Guglielmo Sirleto auch einen gewissen „Amaltheus” als Zensor ausmachen. Mit Letzterem musste Giovanni Battista Amalteo gemeint gewesen sein. ${ }^{530}$ Die Zensuren zu Sigonios Werk stellen nicht nur wegen ihrer philologischen, sondern besonders wegen ihrer inhaltlichen Anmerkungen zur Historia Bononiensis ein zentrales Fragment in der Rekonstruktion der Arbeiten der Indexkongregation gegen die Zenturiatoren dar. Sie erlauben es, die bislang unbeachtete Rolle der kurialen Widerlegungsarbeiten gegen die Zenturiatoren bei der Entstehung von Cesare Baronios Annales Ecclesiastici zu berücksichtigen. Die erhaltenen Dokumente der Zensuren an der Historia Bononiensis zeigen, dass insbesondere Sirleto mit Sigonios Vorhaben nicht einverstanden war. Sigonio legte eine Geschichte Bolognas und des mittelalterlichen Italien vor, ohne dabei das Papsttum als legitime Herrschaftsinstanz anerkennen zu wollen. Deshalb mahnte der Vorsteher der Indexkongregation Sigonio davor, dass eine Geschichte Bolognas ohne Beachtung der res sacra anhand den Ecclesiasticos authores nicht geschrieben werden könne. Sigonio verteidigte dagegen seine Historia mit der Begründung, dass er kein Ecclesiasticus und damit kein Kirchenhistoriker sei. Damit wollte sich Sigonio einer Verpflichtung, sowohl die res Ecclesiasticae als auch die Ecclesiasticae orationes $\mathrm{zu}$ behandeln, entziehen. ${ }^{531}$ Darüber hinaus kritisierte Sirleto an Sigonios Umgang mit der Geschichte Bolognas, dass seine Historio Bononiensis in Widerspruch zur Heiligen Schrift stehe. Sigonios Darstellung Bolognas als ein sich selbständig entwickelndes regimen ohne die Unterstützung des Papsttums glich für den gelehrten Kurienprälaten Sirleto der im Johannes-Evangelium tradierten Verurteilung Jesu durch die Juden, da diese ihm vorwarfen, dass „er sich selbst zu Gottes Sohn gemacht habe“ (Jh 19, 7). Die Formulierung se Regem facit entspräche damit einer kaiserlich orientierten Geschichtsschreibung, auf die es Sigonio hauptsächlich ankam. Demzufolge fielen die juristischen Streitigkeiten um das Regnum Italiae in Sigonios Beschreibung der ottonischen Verwaltung des Königreichs Italien in den Zuständigkeitsbereich des Kaisers und nicht den der Päpste. ${ }^{532}$ Sirleto entgegnete dieser Annahme, dass „diese doch dem Papst zustünden“ und wies Sigonio darauf hin, dass dieser Teil seiner Historia ansonsten ausgelassen werden müsse. ${ }^{533}$

530 Sigonio, Opera omnia, III, S. 331; McCuaig 1989, S. 252.

531 Sigonio, Opera omnia, III, S. 333; BAV, Vat. lat. 6207, f. $97 v$.

532 Sigonio, Opera omnia., III, S. 334.

533 Ibid., S. 61, 339. 
Die Eingriffe in Carlo Sigonios Historia Bononiensis seitens der Indexkongregation mussten auf der Grundlage eines spezifisch kirchenhistoriographischen Verständnisses der Entstehungsgeschichte des Kirchenstaates seit der Konstantinischen Schenkung ausgearbeitet worden sein. Die Zensuren Kardinal Ugo Boncompagnis zum Abschnitt über den 1158 in Roncaglia stattgefundenen Reichstag, an welchem auch Juristen aus Bologna teilnahmen, lassen den Einfluss, den die Correctores Romani auf die Arbeiten der Indexkongregation gehabt haben müssen, erkennen. Die Correctores hatten bereits die kirchenhistoriographische Auslegung kaiserlicher Schenkungshandlungen in das kanonische Recht eingerahmt, weshalb Ugo Boncompagni Sigonio daran erinnerte, dass es die Entscheidung der Bologneser Juristen war, die den imperialen Anspruch Kaiser Friedrichs I. auf die Verwaltung italienischer Städte billigten. Dennoch schlug Boncompagni vor, dass eine solche Aussage verschwiegen werden sollte. Ansonsten würde sie Zweifel an der Gültigkeit von Schenkungen sowohl fränkischer als auch sächsischer Kaiser an das Papsttum in der Nachfolge Kaiser Konstantins d. Gr. hervorrufen. ${ }^{534}$ Zwischen 1570 und 1571 - also zeitnah zu der offiziellen Einberufung der Indexkongregation - setzten sich die Correctores Romani selber damit auseinander, wie die Konstantinische Schenkung in der Neuedition des Decretum Gratiani rechtsgültig verteidigt werden sollte. Demgemäss enthielt das vierzehnte Kapitel der Distinctio 96 in dem 1582 gedruckten Decretum ein langes dictum Correctorum, das anlässlich der am 14. März 1571 gehaltenen Congregatio generalis verfasst wurde. ${ }^{535}$ In einer Randnotiz wird nämlich erwähnt, dass die Kardinäle Boncompagni und Sirleto an dieser Sitzung der Correctores teilnahmen. Die zum dictum Correctorum angefertigten Notizen halten ferner fest, dass ein Mitglied der Correctores dieses capitulum verteidigen sollte. ${ }^{536}$ Das dictum kann dem Juristen Thomás de Taxaquet zugeschrieben werden, da sich seine Erläuterungen zu diesem zentralen Kapitel in einer weiteren Handschrift befinden, die ebenfalls zum Material der Correctores Romani gehört haben musste. ${ }^{537}$ Die Verteidi-

534 BAV, Vat. lat. 6207, f. 74r: „Videtur tollenda illa sententia lata super civitatibus Italiae pro Imperatore, cum sit contra donationes factas Sedi Apostolicae“. Vgl. auch Sigonio, Opera omnia, III, S. 343; McCuaig 1989, S. 254.

535 Dieses Kapitel ist eine Fortführung des capitulum 11, welches die Untertanenpflicht der Kaiser gegenüber den Päpsten deklariert. Vgl. Corp. iur. can., D. 96, c. 11. Vgl. dann auch ibid., c. 14: Constantinus imperator quarta die sui batismi privilegium Romanae ecclesiae Pontifici contulit, ut in toto orbe Romano sacerdotes ita hunc caput habeant, sicut iudices regem. In eo privilegio ita inter cetera legitur: 'Utile iudicavimus una cum omnibus satrapis nostris, et universo senatu optimatibusque meis, etiam et cuncto populo Romanae gloriae imperio subiacenti, ut sicut B. Petrus in terris vicarius Filii Dei esse videtur constitutus, ita et Pontifices, qui ipsius principis apostolorum gerunt vices, principatus potestatem amplius quam terrena imperialis nostrae serenitatis mansuetudo habere videtur, concessam a nobis nostroque imperio obtineant, eligentes nobis ipsum principem apostolorum vel eius vicarius firmos apud Deum esse patronos“ CIC, D. 96 , c. 14 gibt somit das überlieferte Constitutum Constantini wieder und deklariert es als Voraussetzung jeder weltlichen Herrschaft.

536 BAV, Vat. lat. 4889, f. 152v, $154 r$.

537 BAV, Vat. lat. 4890, f. 34r-35r. 
gung der Konstantinischen Schenkung auf der Grundlage der Stelle im Decretum Gratiani fiel aber ungenügend aus. Sommars Einschätzung, Taxaquets Erläuterungen seien eine „art of saying nothing”, trifft zu, denn der spanische Kanonist hatte eine dicta Correctorum verfasst, die weder eine Annahme der Magdeburger Zenturiatoren bestätigte noch die Stellung der Kurie dazu festlegte. ${ }^{538}$ Letztendlich erlaubten es auch Taxaquets dicta Correctorum, kaiserliche Schenkungen in ihrem kirchenhistoriographischen Zusammenhang willkürlich interpretieren zu dürfen. Carlo Sigonio hatte die Konstantinische Schenkung, wenn auch nur beiläufig, in seiner Historia Bononiensis somit als „Glaube frommer Menschen” beschreiben können. Guglielmo Sirleto wendete gegenüber dieser Wortwendung ein, dass sie einerseits nicht angemessen gewählt sei. Andererseits schlug Sirleto vor, dass Sigonio die Schenkung Konstantins d. Gr. als multi probatae fidei scriptores tradiderunt betrachten solle. ${ }^{539}$ Die Konstantinische Schenkung konnte kirchenhistoriographisch somit allein auf der Grundlage ihrer kirchenrechtlichen Überlieferung bestätigt werden.

Guglielmo Sirletos Zensuren an der von Sigonio verfassten Historia Bononiensis setzten sich auch mit Stellen auseinander, in denen der Gelehrte aus Modena auf die kirchenhistoriographische Tradition der caeremoniae einging. An diesen Stellen der Historia Bononiensis lässt sich das Bestreben Sirletos, die Congregatio Germanica von ihren Arbeiten gegen die Magdeburger Zenturien abzulösen und diese Widerlegungsarbeit nach 1576 vollständig in die Aufgabenbereiche der Indexkongregation überführen zu wollen, nachweisen. Aus den Protokollen der Congregatio Germanica geht hervor, dass die vorhin erwähnten, dogmatischen Widerlegungsarbeiten Diego de Ledesmas und Francisco Torres' in denselben Zeitraum fielen, in welchem das römische Collegium Germanicum durch Papst Gregor XIII. die grösste Förderung erhielt. Die Kardinalskongregation wollte sich damit der Stosskraft, welche das unter Julius III. eingerichtete Jesuitenkolleg zur Entsendung von Priestern nach Deutschland ausstrahlte, bedienen. Ferner zeigen die ab 1573 dokumentierten Entscheidungen der Congregatio Germanica, dass die Kongregation mehrmals in die inneren Strukturen des Jesuitenkollegs eingriff. Ein zentrales Anliegen der Congregatio Germanica kam in der Sitzung vom 7. September 1574 zum Vorschein, als sich ihre Kardinäle damit befassten, ob sie die Studenten des Jesuitenkollegs an Gottesdiensten ausserhalb des Collegio teilnehmen lassen sollten. Aus dem entsprechenden Sitzungsprotokoll geht hervor, dass die Gestaltung der Gottesdienste (ceremonias et ritum) innerhalb des Kollegs vom römischen Ritus abwich. ${ }^{540}$ Es ist dieses Problem der Angleichung verschiedener Gottesdienste an den römischen Ritus, das auch in den Zensuren Guglielmo

538 Sommar 2002, S. 80.

539 BAV, Vat. lat. 6207, f. 98r; Sigonio, Opera omnia, III, S. 334.

540 Schwarz, Briefe und Akten, II, S. 97: „Ineatur ratio mittendi alumnos collegii Germanici dictis festis ad ecclesias, ut maiorum missarum et horarum vespertinarum celebrationi intersint ac ceremonias et ritum ecclesiae Romanae addiscant. De hoc agi posse cum rectore collegii Germanici“. 
Sirletos zu Sigonios Historia Bonnoniensis auftritt. Sirleto wies Sigonio darauf hin, dass die im Jahr 590 gesungenen Litaneien Gregors d. Gr. anlässlich des Markusfestes in Bologna als Caerimoniae antea institutae, \& per traditionem Apostolorum zu beschreiben seien. Er begründete dies damit, dass die Häretiker darüber schweigen würden. ${ }^{541}$ Der Präfekt der Indexkongregation sah demnach in Carlo Sigonios Historia Bononiensis ein Werk, das eine erfolgreiche Widerlegung der Magdeburger Zenturien versprach. Aus der historiographischen Darstellung Sigonios trat allerdings nicht die für Sirleto wichtige Einsicht hervor, dass die Geschichte Bolognas ein vortreffliches Musterbild der Angleichung an ein römisch-kuriales Bezugssystem des Zeremoniells schlechthin präsentierte. Die Geschichte Bolognas war dennoch als Vorlage einer Kirchengeschichte dienlich, denn anhand dieser würde sich die Übereinstimmung der Stadtentwicklung mit der Entfaltung des römischen Ritus beispielhaft zeigen lassen.

Guglielmo Sirleto hatte damit im Gegensatz zu den Correctores Romani die Kirchenhistoriographie innerhalb eines vom römischen Ritus vorgegebenen Rahmens verstanden und fasste sie nicht zwangsläufig allein gemäss der Vorschrift des kanonischen Rechts auf. Dieser Rahmen eignete sich deshalb als geeignete Grundlage, da mit diesem auch der päpstliche Machtanspruch in einer Auslegung der translatio imperii gezielt verteidigt werden konnte. Der Gelehrtenprälat Sirleto stellte damit einen Weg einer Widerlegung protestantischer Kirchengeschichtsschreibung vor, der den Forderungen der Correctores Romani gerecht wurde. Guglielmo Sirletos Bestreben, verschiedene Diözesanriten, aber allen voran den Ritus Bolognas, an den römischen Ritus anzugleichen, wurde ebenfalls von Ugo Boncompagni begrüsst. Der später zum Papst gewählte Kardinal war nämlich mit Carlo Sigonios Umgang sowohl mit dem Privilegium Theodosii als auch mit dem liturgischen officium des heiligen Petronius, des Stadtheiligen Bolognas, unzufrieden. ${ }^{542}$ Eine anonym erhaltene Kritik zu dem 1571 erschienenen De Regno Italiae Carlo Sigonios zeigt, dass Sigonios Geschichtsschreibung mit den Forderungen der römischen Kurie nicht kompatibel war. Der anonyme Autor dieser Kritik bemerkt nämlich, dass Sigonios De Regno Italiae bei der Darstellung der Rolle des Papsttums als Heilserlösung Italiens offensichtliche Mängel aufweist:

Nel principio della historia dice la ruvina della Italia esser stata causata dai Papi. Et oltre che questa è opinione del Machiavelli ne discorsi, è pericola ne tempi d'hora. Oltre che il papato, e questo et sempre, ha chiamato alla defensione di quella contra barbari, et Franza et altri

541 Sigonio, Opera omnnia, III, S. 336.

542 Fasoli 1972-1973, S. 69-94; Bastia 1993, S. 99-113; Prodi 2005, S. 298-299. Zu Sigonio und dem liturgischen officium des heiligen Petronius in Bezug auf die Rolle des Bischofs von Bologna, Gabriele Paleotti, vgl. v. a. Prodi 1959/67, Bd. 2, S. 252-257; Ditchfield 1995, S. 63-65. 
Regni. Et almeno l'esser italiano el Sigonio, toccava a lui mantener la riputazione del papato; per che altro bene non è in essa, che questa Santa Sede. Et questa ragione politica doveva certo fare più cauto el Signor Sigonio. ${ }^{543}$

Die entscheidende Strategie für eine Kirchengeschichte, die den kurialen Massstäben gerecht wurde, musste damit zuerst den historiographischen Widerspruch der Unveränderlichkeit des sich ständig im Wandel begriffenen Papstamtes lösen. Erst dann konnte auch dessen Rechtsgültigkeit, wie sie im neuen Corpus Iuris Canonici formuliert wurde, kirchenhistoriographisch begründet werden. Während somit die beiden Jesuitentheologen Diego de Ledesma und Francisco Torres für die Congregatio Germanica eine kontroverstheologische Grundlage solcher historiographisch unveränderbarer Momente aufbereiteten, arbeitete die Indexkongregation unter der Leitung Guglielmo Sirletos an einer authentischen Erzählung der Entwicklung der römischen Kirche, die sich aus den Zensuren am historiographischen Werk Carlo Sigonios ableiten liessen. Guglielmo Sirleto hatte damit innerhalb der Kurie ein spezifisches Neuverständnis der Kirchenhistoriographie gestiftet. Die Ecclesiastica Historia wurde nicht mehr länger bloss als eine Neufassung einer Profanerzählung verstanden, sondern vielmehr als eine Erzählform, aus welcher ein dynamischer Übergang der doctrinae in die caeremoniae hervorging. Damit wurde folglich eine Auslegung der translatio imperii in die Wege geleitet. Diese entscheidende Einsicht, dass die Kirchengeschichtsschreibung einen solchen für das Papsttum und die römische Kirche zentralen Übergang angemessen darzustellen hatte, fiel mit der theologisch begründeten Dogmatik nicht nur des Papstamtes, sondern auch der gesamten Kurie zusammen. Die Erzählung einer solchen Entwicklung der Romana Ecclesia forderte demgemäss eine enge Zusammenarbeit zwischen Indexkongregation und Congregatio Germanica. Erst dadurch liessen sich die doctrinae mit den caeremoniae in Übereinstimmung bringen. Die Idee von Cesare Baronios Annales Ecclesiastici kann als Produkt dieser Zusammenarbeit der beiden Kongregationen betrachtet werden.

\subsection{Die Idee der kirchenhistorischen Annales}

Am 16. Mai 1577 bat Cesare Baronio den Präfekten der Indexkongregation um die Erlaubnis, die Ecclesiastica Historia der Magdeburger Zenturiatoren lesen zu dürfen:

543 BAM, R 109sup., f. 148r. Vgl. auch den Brief Sigonios an Gian Vincenzo Pinelli vom 2. Mai 1573 in ibid., f. 147r: „Che nel principio dell'historia si dica, la rovina dell'Italia esser stata causata dai papi, questo si nega esser né detto né significato“. Sigonio vermutet ferner, dass die avertimenti „von einem Kardinal kämen, der aber wenig Wissen und wenig Kenntnis, aber grosse Leidenschaft zeige“. (ibid., f. 149r.) Es ist unwahrscheinlich, dass es sich dabei um Sirleto gehandelt hat, wie McCuaig 1989, S. 258, vermutete. 
Perché desidero con l'aiuto d'Iddio et favor di Sua Signoria incominciar da capo a rescrivere l'historia ecclesiastica et ponervi l'ultima mano, la prego se degni oprar con l'illustrissimo et Reverendissimo Card. di Pisa di farmi dar licentia di posser tenere et leggere le Centurie, et che me siano dal'instesso imprestate, et parendogli domandar ancor licentia di posser tenere et leggere con correggere dove bisogni la Bibliotheca santa, et questo per la commodità di molti autori in quella insieme ragunati, quali altrove con difficultà si haveriano..$^{544}$

Innerhalb der Forschung gilt dieses Briefschreiben als Beleg dafür, dass der Oratorianer Baronio ab diesem Zeitpunkt seine conferenze sulla storia ecclesiastica angesichts ihrer Drucklegung innerhalb des Oratoriums zu redigieren begann. ${ }^{545}$ Der Brief Baronios wirft aber im Spiegel der vorhin dargelegten Entwicklungen der kurialen Widerlegungsarbeiten gegen die Magdeburger Zenturien mehr Fragen auf, als er beantwortet. Im ersten Teil der vorliegenden Untersuchung konnte gezeigt werden, dass sich die Tätigkeit Baronios innerhalb der Priesterversammlungen der Oratorianer hauptsächlich auf Predigten zu ausgewählten Heiligenviten konzentriert haben muss, die Baronio dann auch an spezifischen Feiertagen im liturgischen Kalender gelehrt haben muss. Es kann sich also in diesem Briefschreiben vom 16. Mai 1577 keineswegs um eine „valutazione più prudente“ gehandelt haben, wie dies Fubini darzulegen versuchte, denn diese Predigten wiesen noch keineswegs einen kirchenhistoriographischen Umgang mit den Heiligenviten auf. ${ }^{546}$

In Baronios Briefschreiben an Sirleto tauchen drei Hinweise auf, die darüber Aufschluss geben, in welchem kulturellen Zusammenhang er die Abfassung seiner Annales Ecclesiastici begann. Den ersten Anhaltspunkt bietet die Übereinstimmung von Baronios Biblioteca sancta mit der Bibliotheca selecta des Dominikaners Sisto da Siena, die erstmals im Jahr der Papstwahl Pius ${ }^{6}$ V. in Venedig gedruckt wurde. ${ }^{547}$ Unmittelbar nach ihrem Erscheinen wurde die Bibliotheca selecta als Referenzwerk für Bibelstudien schlechthin angesehen. Sie gilt daher auch als eine der ersten Enzyklopädien hermeneutischer Bibelexegese nach Trient. ${ }^{548}$ Die Erwähnung der Bibliotheca selecta in Cesare Baronios Briefschreiben ist deshalb von zentraler Bedeutung, da sie die Arbeiten

544 BAV, Vat. lat. 6192, II, f. 634r-635r (Hervorhebung d. Verfassers); Mercati 1937, S. 275; Paschini 1923, S. 200-201. Der Kardinal Scipione Rebbiba, der hier ebenfalls genannt wird, starb allerdings am 24. Juli desselben Jahres, in welchem Baronio seinen Brief an Sirleto verfasste. Zu diesem zentralen Mitarbeiter des Santo Uffizio, der ab dem Pontifikat Gregors XIII. nach einer Definition eines „stile dell'Inquisizione“ arbeiten sollte, vgl. insbes. DBI 2016, Bd. 86; DSI, Bd. 3, S. 1303-1304.

545 Zen 1994, S. 69-71; idem 2008, S. 26-28.

546 Fubini 2003, S. 363.

547 Nach dem Tod des Dominikaners erfuhr sie eine Neuauflage 1575 in Frankfurt und ein Jahr später in Köln. Zwischen 1540 und 1550 hatte Sisto da Siena zu Ambrogio Catarino Politis Doktrin der Prädestination gepredigt, um die häretische Position darüber non sine audientium plausu, ac perturbatarum mentium fructu zu widerlegen. Er gilt daher als einer der wichtigsten Anhänger Politis und seines theologischen Denkens. Vgl. hierzu v. a. Montgomery 1963, S. 221-222; Caravale 2007, S. 296-298. Zusammenfassend auch DThC 1914, XIV/2, S. 2238.

548 Für eine der treffendsten Beschreibungen dieses Werkes vgl. insbes. Du Pin, A new history of ecclesiastical writers, S. 98. 
Guglielmo Sirletos an der Herausgabe einer neuen Vulgata-Edition und die damit verbundene Einführung einer spezifisch bibelhermeneutischen Theologie in die kurialen Widerlegungsarbeiten der Congregatio Germanica andeutet. Diesen Neuzugang zur Widerlegung der Zenturien griff bereits der Jesuit Roberto Bellarmino in seinen ab 1576 im Collegio Romano gehaltenen Vorlesungen auf, die er später als Controversiae zwischen 1588 und 1593 herausgab.

Bellarmino, der bis 1576 den Lehrstuhl für Theologie an der Universität von Louvain innehatte, bat Sirleto in einem Briefschreiben vom 1. Mai 1575 darum, Sirletos Anmerkungen zu den von Lorenzo Valla und Erasmus verfassten Annotationen zu dem von Plantin gedruckten Neuen Testament lesen zu dürfen. Bellarmino beabsichtigte, die perniciose annotazioni Vallas und des Erasmus zu widerlegen. Aus diesem Schreiben an den Präfekten der Indexkongregation darf vermutet werden, dass es Guglielmo Sirleto war, der die Initiative ergriff, den Jesuiten Bellarmino in die kurialen Arbeiten zur neuen Vulgata einzubinden. Bellarmino verfolgte die Widerlegung der Annotationen Vallas und des Erasmus sowohl auf der Grundlage des Tridentiner Dekrets zur Heiligen Schrift als auch anhand Sisto da Sienas Andeutungen in der Bibliotheca selecta zu den letzten Kapiteln im Buch Esther, die zu Apokryphen erklärt wurden. ${ }^{549}$ Am 26. November 1576 erhielt Bellarmino am Collegio Romano die cathedra für Theologie. Am selben Tag hielt der früher in Louvain tätige Theologe seine Inauguralvorlesung, die als Vorrede in seine ab 1588 gedruckten Controversiae aufgenommen wurde. Bellarminos Vorlesung von 1576 bildete den Auftakt seiner ersten controversia generalis, womit der Jesuitentheologie die zentrale Bedeutung der Kontroverstheologie anhand dogmatischer Fehler der Protestanten aufzeigte. Die abgedruckte Vorrede im ersten Band der Controversiae stellt einen programmatischen Entwurf Bellarminos für eine Kontroverstheologie dar, die Motta treffend als eine tassonomia dell'errore bezeichnete. ${ }^{550}$

549 Le Bachelet 1911, S. 90-94, 92-93: „[...] venio nunc ad quaestiones meas, quarum prima ac praecipua illa est, quid sibi Tridentina synodus voluerit cum 4 a sessione latinam et vulgatam editionem, pro authentica habendam essere decrevit. [...] Altera quaestio est an codices hebraei quos nunc habemus, integri et incorrupti, an potius vitati ac depravati existimandi sunt. [...] Iam tertia quaestio de 70 interpretum versione exoritur; quidam enim hebraeae grecaeque linguae peritissimi tantam offendunt in graecis Scripturae sacrae codicibus diversitatem, sive constructionis formas, sive puritatem atque elegantiam orationis spectare velimus, ut nulla ratione videatur unus atque idem esse potuisse omnium divinorum librorum interpres [...]. Postrema quaestio est, an septem ultima capita libri Esther sacra atque canonica habenda sint, nec ne“. Zur Überführung Bellarminos von Louvain nach Rom ab 1574 vgl. die Briefkorrespondenz des Jesuitengenerals Mercurian in MPSI, IV, S. 530; Le Bachelet 1911, S. 101-102.

550 Motta 2005, S. 164. Die Controversiae Bellarminos befinden sich in ihrer handschriftlichen Überlieferung in BAV, Vat. lat. 6613-6617. Einzelne Teile, wie die Traktate De verbo Dei, De summo Pontifice und De clericis finden sich in BAM, D 180inf. Die folgenden Ausführungen stützen sich hauptsächlich auf die Praefatio in disputationes de controversiis christianae fidei adversus huius temporis haereticos in Fèvre, Roberti Bellarmini politiani S. J. opera omnia, I, S. 53-62. Die deutschen 
Die „Kampfpunkte des Glaubens“, die Bellarmino „gegen alle Ketzer dieser Zeit“ zusammenfassend darlegen möchte, verlangen nicht nur die Kenntnis „verschiedener Wissenschaften und Sprachen, sondern auch des ganzen Altertums“ sowie „aller Geschichten und Zeiten“. ${ }^{551}$ Bellarmino legt in seinen Ausführungen die utilitas der Kontroverstheologie (controversiae) als eine Disziplin aus, die eine erfolgreiche Widerlegung ketzerischer Glaubensartikel ermöglicht:

Und damit nicht allenfalls unsere Worte einigen missfallen, welche die Pest der Ketzerei für geringfügig halten, weil sie dieselbe nach den gewöhnlichen Lastern und Sünden bemessen, deren Reinigung und Heilung durch das Sakrament der Busse sie täglich bemerken, so will ich über die Wichtigkeit der Ketzerei reden, nicht alles, was sich sagen lässt, auch nicht das meiste [...] sondern nur weniges, was teils notwendig, teils hier hinreichend scheinen kann. [...] Nur dies einzige will ich bemerken, dass die Gottlosigkeit der Ketzerei alle anderen Verbrechen und Schandtaten an Schwere so weit übertrifft als die Pest an Schrecklichkeit und Fürchterlichkeit die gewöhnlichen Krankheiten. [...] Dasselbe, was den Leibern die Pest, ist auch die Ketzerei nur in den Seelen. Das erste Gnadengeschenk, welches wir vom himmlischen Vater, bei der Umwandlung und Rechtfertigung, empfingen, die erste Bewegung des wiederauflebenden Herzens, der erste Sinn des geistlichen Lebens ist ohne Widerrede und Zweifel der Glaube [...] Kann nun eine verderblichere und pestilenzialischere Seuche gedacht werden als die Ketzerei, da sie sogleich das Innerste der Seele angreifet, bei Entreissung der Gnadengaben bis auf den Grund dringt und jenen Anfang des göttlichen und himmlischen Lebens tötet? Der Grund des geistlichen Gebäudes ist, nach dem Bekenntnis und der Meinung aller, der Glaube. ${ }^{552}$

Auf der Suche nach einem geeigneten Heilmittel gegen die pesta haereseos stellt für Bellarmino die Kontroverstheologie somit eine Art Therapie dar, bei welcher die Ketzerei als kirchenhistoriographisches Phänomen enthüllt wird. Der Glaube bildet dabei die Grundlage einer jeden Widerlegung der Häresie. Gleich einem Arzt solle der Theologe anhand der Heiligen Schrift und der Kirchenväter die Symptome dieser Krankheit erkennen und entsprechende Heilmittel zur Genesung der Seele anbieten. ${ }^{553}$ Bellarminos Controversiae stellen daher den ersten erfolgreichen Versuch dar, wie eine Historiographie des menschlichen Heilsgeschehens in einen theologischen Mantel eingekleidet werden sollte. Erst dadurch kann die veritas von häretischen Fehlern unterschieden werden. Häretische Glaubensartikel lösen sich somit in einem solchen kirchenhistoriographischen Trennungsverfahren auf. Eine solche

Übersetzungen daraus, falls nicht anders vermerkt, basieren auf der jüngsten Edition der Disputationes: Gumposch 2012, Bd. 1, S. XI-XVII.

551 Bellarmino, Praefatio, in Fèvre, Roberti Bellarmini politiani S. J. opera omnia, I, S. 53: „Arduum sane opus, et certe, mea quidem sententia, ejusmodi, ut si nn leviter et perfunctorie, sed accurate, ac ut rei dignitas, et difficultas exigit, tractandum sit: scientiam prope infinitam, non modo variarum disciplinarum, et linguarum, sed etiam totius antiquitatis omnium historiarum, et temporum requirat“" (BAV, Vat. lat. 6613, f. 1r).

552 Ibid., S. 54-55; BAV, Vat. lat. 6613, f. 1v-2r; BAM, D 180inf., 1v-2r.

553 Ibid., S. 57-58. 
Reinigung kann aber gemäss dem Jesuiten Bellarmino allein mittels einer genauen Rekonstruktion der Unterschiede zwischen häretischen Glaubensartikeln und dem apostolischen Glaubensbekenntnis - dem Symbolum Apostolicum - erreicht werden. Dem Gläubigen sollte damit gezeigt werden, in welcher Weise die katholische Kirche über alle diese „Schwärme“ (copii mores) schliesslich triumphiere. ${ }^{554} \mathrm{Im}$ Glaubensbekenntnis der Apostel ist für Bellarmino somit die reinste Formulierung eines jeden kirchlichen Glaubensbekenntnisses gespeichert. Gemäss diesem Bekenntnis der Apostel könnten die haeretici huius temporis innerhalb einer paradigmatischen Konstellation zwischen Wahrheit und fehlerhaftem Glauben bekämpft und bezwungen werden. Diesen paradigmatischen Kampf wird Bellarmino mehrmals in seinen Controversiae am Collegio Romano aufgreifen. Schon ein Jahr nach der Inauguralvorlesung eröffnete Bellarmino seine kontroverstheologischen Vorlesungen über den Summo pontifice. Vier Jahre danach folgten die controversiae über die Doppelgestalt Christi. ${ }^{555}$ Cesare Baronios Erwähnung von Sisto da Sienas Bibliotheca sancta muss folglich als ein Hinweis auf eine besondere Stelle in Bellarminos Controversiae beurteilt werden. Nach Bellarminos Kontroverstheologie galt die $\mathrm{Bi}$ bliotheca sancta als eigentliches Referenzwerk, mit deren Hilfe diejenigen Ketzereien erschlossen werden konnten, welche die alttestamentlichen Bücher erster Ordnung bekämpften und die auch in Bellarminos controversia zum Gotteswort explizit erwähnt werden:

Obgleich aber in der katholischen Kirche das Ansehen der aufgezählten Bücher, unter Übereinstimmung aller Rechtgläubigen, immer aufrecht stand, so fehlte es doch nicht an Ketzern, welche trachteten, denselben Büchern alles Ansehen geraden Weges zu entziehen. Weil aber Sixtus (Senensis) am Ende seiner Bibliothek jene Ketzereien, welche durch den Eifer unserer Vorfahren längst ausgelöscht und begraben waren, in unserer Zeit wieder dargestellt hat, so kann ich mich so kurz als möglich mit diesen alten Feinden, oder vielmehr Schatten von Feinden, befassen..$^{556}$

Baronios Bitte an den Kardinalpräfekten Guglielmo Sirleto, die Bibliotheca selecta lesen und gegebenenfalls an einigen Stellen korrigieren zu dürfen, enthält die weiter-

554 Ibid., S. 59-60.

555 BAV, Vat. lat. 6614, f. 1r-2r (Praefatio in controversiam de Summo pontifice); BAV, Vat. lat. 6617, f. 322r-v (Praefatio de duplici genere hostium divinitatis Christi). Die erste Praefatio ist auch in BAM, D 180inf., f. 91r-94v erhalten. Vgl. zu diesem kirchenhistoriographischen Paradigma in Bellarminos Controversia insbes. Ryan 1936, S. 152-154; Polman 1932, S. 513; Dietrich 1999, S. 70-72; Motta 2005, S. 168-169.

556 Bellarmino, Controversiarum de Verbo Dei, in Fèvre, Roberti Bellarmini politiani S. J. opera omnia, I, S. 75: „Et si vero in Ecclesia Catholica Orthodoxorum omnium consensione enumeratorum librorum semper viguit auctoritas; non defuere tamen haeretici, qui libris iisdem omnem prorsus auctoritatem detrahere niterentur. Quoniam autem illas eorum haereses majorum nostrorum diligentia jamdudum excinctas ac sepultas, rursum hoc nostro saeculo Sixtus Senensis in extrema sua Bibliotheca stylo prasecutus est, quam brevissime potero ab antiquis istis non tam hostibus, quam umbris hostium me expediam“. 
führende Erklärung, dass der Oratorianer seine Korrekturen und Ergänzungen per commodità di molti autori anfertigen möchte. Dabei musste es sich um ein Projekt gehandelt haben, das sich erst in seiner Frühphase befand. Ausserdem deutete Baronio damit an, dass er sein Vorhaben ausschliesslich im Zusammenhang mit der Thematik der heiligen Bücher anfertigen möchte, und zwar auf ähnliche Weise wie Bellarmino seine Vorlesungen zur Kontroverstheologie gestaltete. ${ }^{557}$ Dass Baronio dabei weiter um die Erlaubnis der Einsicht in die Magdeburger Zenturien bat, die ab 1559 in den Index aufgenommen wurden, verrät ausserdem, dass er ein Referenzwerk vorlegen wollte, das dieselbe Autorität wie Sisto da Sienas Bibliotheca selecta besitzen sollte. Dieses vom Oratorianer Baronio angefertigte Referenzwerk sollte der Indexkongregation für eine neu zu schreibende Historia Ecclesiastica dienen.

Cesare Baronio wollte somit die Historia Ecclesiastica „von Anfang an neu schreiben“ (da capo a rescrivere l'historia ecclesiastica). Damit können aber keinesfalls seine eigenen Predigten innerhalb des Oratoriums gemeint gewesen sein. Das Prädikat rescrivere muss sich hier nämlich auf das bereits in den Zensuren zu Carlo Sigonios Historia Bonnoniensis beobachtete Verfahren der Indexkongregation beziehen, bei welchem historiographisch unsaubere Stellen gereinigt und in eine für die Kurie passende Form überführt wurden. ${ }^{558}$ In der Vorrede zum ersten Band seiner Annales Ecclesiastici unterscheidet Baronio die Gattung der historia ecclesiastica von derjenigen „kirchlicher Jahrbücher”. Während erstere Disziplin von zeitgenössischen Handlungen und den dahinterliegenden Bedeutungen berichte, suche eine Annalistik bei der Dokumentation der monumenta jeden Jahres die veritas als sancte (heilsam), pure (rein) und sincere (ehrlich) darzulegen. Erst in der Annalistik könne man der majestas ac gravitatem maxime Ecclesiasticam gerecht werden. ${ }^{559}$ Der Bibliothekar Aniello Sanvito hat in einem Brief an Sirleto, der auf dasselbe Datum wie Baronios Schreiben an den Kardinal datiert ist, dieses Verfahren der expurgatio seitens der Indexkongregation näher bestimmt und gleichzeitig auch die Kirchengeschichte darunter aufgeführt:

557 Ibid., S. 74-75.

558 Für solche sprachlichen Umformulierungen des Lateinischen in Sigonios Historia Bonnoniensis vgl. dann McCuaig 1989, S. 256-257.

559 Baronio, Apparatus, S. 396: „Annalium contra scriptor res antiquas, ut plurimum quas sua non novit aetas, easdemque per annos singulos monumentis commendet. Nos autem, quoniam non tantum res antiquas, sed Ecclesiasticas potissimum petractamus, in quibus non solum, ut in caeteris historiis, ipsa veritas primum sibi vendicare locum debet, sed vel latum unguem ab ea recessisse, religio est: hanc ob causam, ne proditae veritatis vel levi saltem suspicione pulsemur, Christianis legibus obsequentes praecipientibus 'Sit sermo verster: est est, non non, quod autem his abundantius est, a malo est': relinquemus historicis Ethnicis locutiones illas per longiorem ambitum periphrasticae circumcundas, orationesque summa arte concinnatas, fictas, ex sententia cuiusque compositas, ad libitumque dispositas; et Annales potius, quam historiam scribemus“. Vgl. auch Mazza 2012, S. 41; Fossati 1938, S. 538-562; Ferrarini 1982, S. 320; Zen 1994, S. 69-70; Pullapilly 1975, S. 147-164; Malesevic 2015, S. 47-48. 
[...] si è detto molto tempo fa che in Roma si attendeva all'espurgazione di molti libri, per darla poi fuori per comune utilità et opera di carità, et saria stata sollevamento di molti librari, quali tuttavia declinano nella povertà, per tener ispedite et perse molte sorte de‘ libri che, o non sono nell'Indice, o si pure in alcuno modo si comprendono esser conforme alle regole dell'istesso, espurgati si promettevano. Così si costumò per il passato, non se seguì poi, et non è qua chi voglia pigliar la fatiga, et dicono sempre aspettar da Roma uno nuovo Indice di tutti li luoghi de‘ libri da espurgarsi. Incominciò a comparirne segno, nel tempo della bona memoria del Magister Sacri Palatii passato, havendo dato alle stampe l'espurgatione delli testi delli consigli di Alessandro et di Decio; ma, morto esso, a fatto si spense in noi la speranza di haverne a vedere più. Di nuovo ci è stato detto che in Roma si seguiti, ma le cose anderanno a lungo, però ci siamo resoluti insieme ad altri librari di qua et abbiamo mandato supplica alli illustrissimi deputati sopra l'espurgatione, se per avventura si potrà haver gratia dell'espurgatione d'alcuni libri, contentandoci che si cavino a nostre spese. Petranto humilmente la prego si degni aiutar col suo favore questa nostra impresa, se però li par giusta. ${ }^{560}$

Unter der Leitung Guglielmo Sirletos hatte sich die Indexkongregation auf die von Tommaso Manriques begonnene Arbeit der Reinigung liturgischer Bücher gestützt, die im Tridentiner Index aufgeführt wurden und vom Maestro del Sacro Palazzo anhand der Kriterien von heiligen Büchern gereinigt wurden. Dasselbe Bedürfnis griff auch die Congregatio Germanica auf. Das von der am 4. Januar 1578 abgehaltenen Sitzung der Kongregation überlieferte Protokoll hielt fest, dass der Kurfürst und Erzbischof von Trier, Jakob III. von Eltz, eine Petition bei der Kongregation eingereicht hatte, um die im Index aufgeführten,,verbotenen Bücher“ nach entsprechenden Kriterien auszuwählen, damit diese dann innerhalb der Kongregation gelesen werden konnten. ${ }^{561}$

Dieses innerhalb der Congregatio Germanica geäusserte Vorhaben erlaubt es, die Entstehung der Idee der Annales Ecclesiastici nicht mehr länger im Umfeld von Filippo Neris Priesterversammlungen zu verorten. Cesare Baronios Absicht, wie er sie dem Gelehrtenprälaten Sirleto schilderte, war eng mit den Arbeiten der Indexkongregation verzahnt. Die Reinigung von Büchern, die in den Tridentiner Index aufgenommen wurden und unter denen sich auch die Zenturien befanden, sollte allen voran den Kurfürsten und Bischöfen für die Verwaltung ihrer Diözesen in Deutschland dienen, damit sie das apostolische Glaubensbekenntnis verteidigen konnten. Die Indexkongregation sah unter der Leitung ihres Kardinalpräfekten Guglielmo Sirleto ihre Arbeit vor allem darin, in Belangen der kirchlichen Diözesanverwaltung, welche die Bischöfe innerhalb ihrer Kongregationen äusserten, dienen zu können. Diese spezifische Tätigkeit der Indexkongregation deckte sich auch mit der Überführung römischer Riten aus dem Breviarium und Missale Romanum in ein-

560 BAV, Vat. lat. 6416, f. 312v-313r.

561 Schwarz, Briefe und Akten, II, S. 113: „De petitionibus archiepiscopi Treverensis. [...] De lectione librorum prohibitorum. Referendum ad S.mum, an placeat, petitam facultatem dare archiepiscopo praefixo certo numero et descripta qualitate eorum, quibus eam impartiri possit quorumque nomina Sti S. scribere debeat, postquam dederit“. 
zelne Diözesen. ${ }^{562}$ Die Widerlegung der Magdeburger Zenturiatoren hatte die Disziplin der Historia Ecclesiastica in den Bereich des Tridentiner Topos von heiligen Büchern gerückt. Dadurch entwickelte sich innerhalb von Sirletos Indexkongregation eine neue Vorstellung der Kirchengeschichte. Die Kirchenhistoriographie sollte nämlich eine stetige Fortführung dieser heiligen Bücher aus der Heiligen Schrift bewerkstelligen. Diese Vorstellung hat die Historia Ecclesiastica als ein Buch aufgefasst, das am Schluss der Kontinuität zwischen liturgischen Büchern und einer neuen Vulgata stand. Die Entstehung der Annales Ecclesiastici des Oratorianers Cesare Baronio muss als Reinigung einer bereits bestehenden Historia Ecclesiastica betrachtet werden, die im Umkreis von Sirletos Indexkongregation zirkulierte und zensiert werden sollte.

\subsection{Die Annales Ecclesiastici als Expurgation der Historia Ecclesiastica}

Ho veduti alcuni fogli del Signor Sigonio [...] et ho notati alcuni advertimenti [...] Vorrei che in questi nostri miseri tempi s'osservasse questo in esaminare bene i libri; et trattati, et levare tutte quelle parole che possono corrompere le anime et etiamdio le orecchie di giovani et putti. ${ }^{563}$

In seinem Brief an Guglielmo Sirleto erwähnt der Oratorianer Baronio die Zenturien daher nur als Referenzwerk und nicht als zentrales Schriftstück, das ihm sein Vorhaben hauptsächlich ermöglicht habe. Mit der historia ecclesiastica bezog er sich auf ein innerhalb der Kurie in Umlauf befindendes Werk, das jedoch erst im Entwurf vorlag. Cesare Baronios weiterer Briefaustauch mit Carlo Sigonio ermöglicht es, das Vorhaben dieser von der Indexkongregation höchst bedenklichen Historia Ecclesiastica näher zu bestimmen. Es handelte sich dabei um ein nie zu Ende geführtes Vorhaben Sigonios, von welchem Baronio selbst in Kenntnis gesetzt wurde. Am 23. November 1579 fragte der Gelehrte aus Modena beim Oratorianer nämlich nach, ob Baronio dazu gekommen sei, die drei Bücher seiner Historia Ecclesiastica zu konsultieren. In diesem Briefschreiben pries Sigonio besonders die Gelehrsamkeit des Oratorianers, weshalb er sich auch ein Urteil von Baronio über sein Werk wünschte:

Gia da molti dì sono ho inteso da molti della molta virtù, \& valore di V.S., \& specialmente dello studio, che ella pone nella notitia delle cose Ecclesiastiche, nel quale ella eccelle. Ond'io l'ho cominciata ad amare con quello affetto, che la virtù medesima si suole amare. Poscia di

562 Die Frage, wie weit die Tätigkeiten des Kardinals und Präfekten der Indexkongregation im Falle Deutschlands tatsächlich gingen, soll für eine weitere Studie aufgespart bleiben: F. Malesevic, Holy Family and Sacred Palace. Es sei aber angemerkt, dass diese ersten Versuche gescheitert sein müssen, zumal in Deutschland die einzelnen Diözesen zu Lebzeiten Sirletos noch ihre eigenen liturgischen Bücher beibehielten. Vgl. in diesem Zusammenhang auch die schwierigen Verhandlungen Sirletos mit Mantua: BAV, Vat. lat. 6182, f. 136r, 138r-v; ivi, Vat. lat. 6190, f. 130r, 157r, 161r, 322r-v.

563 BAV, Vat. lat. 3455, f. 9r-14v, anonymes Schreiben vom 14. Juni 1578. 
nuovo mi si è aggiunto un vincolo non dirò più stretto [...] Poichè io per lettere d'amici ho inteso, come ella per sua cortesia mi ama, \& loda le mie scritture contutto che poco meritevoli del testimonio suo. [...] Onde io sono stato sforzato a scriverle queste quattro parole per ringratiarla, \& farle sapere, che tutto ciò mi è stato sopra modo grato. Perciocchè premio maggiore non aspetto delle mie fatiche, che un testimonio così honorato, come è il suo. Per la qual cosa voglio pregarla a ricevermi come suo, ò come dedito alle medesime arti, ò come beneficato da lei, ò come naturalmente amico a tutti pari suoi. Desidero sapere, se per avventura Monsig. Illustrissimo Sirletto (sic!) le havesse mostrati tre libri più tosto bozzati, che scritti da me dell'historia Ecclesiastica, \& ciò che ne le pare liberamente, come tra Christiani, \& amici si de‘ procedere, \& all'incontro le offerisco ciò che io posso per se, \& per gli amici suoi. ${ }^{564}$

Die in diesem Briefschreiben erwähnten,,kirchlichen Angelegenheiten“ (cose Ecclesiastiche) verweisen darauf, dass Sigonio ein weit umfangreicheres Werk als nur eine Kirchengeschichte im Sinne hatte. Carlo Sigonios Brief stellt auch deshalb ein wichtiges Fragment für eine kritische Rekonstruktion der Entstehung von Cesare Baronios Annales Ecclesiastici dar, da Sigonio in diesem den Oratorianer als seinen Gewährsmann erwähnt. Sigonio erhoffte sich eine positive Rückmeldung Cesare Baronios, die zu einer erfolgreichen Drucklegung der drei Bücher seiner Historia Ecclesiastica beitragen würde. Außerdem hoffte Sigonio, sich damit einen weiteren Schlagabtausch mit Sirleto und der Indexkongregation ersparen zu können. In der Biblioteca Vallicelliana finden sich drei Werke Sigonios, die zum Bestand von Baronios eigener Büchersammlung gehörten. Hätte Sigonio seine Historia Ecclesiastica tatsächlich zum Druck gebracht, hätte sie wahrscheinlich die beiden weiteren in diesem Inventar aufgezählten Werke - sein De antiquo iure populi romani von 1574 und seine Historiarum de occidentatili imperio libri XX von 1578 - vervollständigt. Die Aufzählung dieser beiden publizierten Abhandlungen in Baronios Bücherinventar ermöglicht eine bislang noch kaum vorgenommene Eingrenzung des Zeitraums, in welchem Baronio erstmals die Idee seiner Annales gefasst haben könnte. ${ }^{565}$

Carlo Sigonio erhielt für sein De occidentali Imperio, mit welchem er wahrscheinlich schon 1575 begonnen hatte, die päpstliche Druckerlaubnis ein Jahr vor seinem erwähnten Schreiben an Baronio. In dieser Abhandlung hatte sich Sigonio der Kaiserzeit zwischen Diocletian und Justinian gewidmet, weshalb er im De occidentali Imperio auch ausführlicher auf die Konstantinische Schenkung einging als in seiner Historia Bononiensis. In Letzterem hatte er den Schenkungsakt nur beiläufig im Zusammenhang mit anderen karolingischen Kaiserschenkungen erwähnt. Sigonio sprach dem Constitutum Constantini - dem Dokument, das die legendäre Schenkung stützte - in Bezug auf den weltlichen Machtanspruch der Päpste aber jedwede Gültigkeit ab. Ein in der Biblioteca Ambrosiana aufbewahrtes und von Si-

564 ACN, XVII, f. 134r; Alberici, Epistolae et opuscula, III, S. 137-138.

565 Sigonio, De antiquo iure populi romani in BVR, S.Borr.G.IV.24. Die Historiarum de occidentali imperio ist im Bücherinventar BVR, Q 72, c. 435r an erster Stelle aufgelistet. Vgl. hierzu insbes. Zen 1994, S. 410; Finocchiaro 2008, S. 252. Vgl. darüber hinaus das Inventarium bonorum vom 2. Juli 1607 in ASR, Auditor Camerae (notaio Domenco Amadeo), prot. 78, c. 2r-7v. 
gonio selbst verfasstes memorandum erlaubt es, den problematischen Umgang Sigonios mit der Konstantinischen Schenkung in seinem De occidentali Imperio zu rekonstruieren. ${ }^{566}$ In diesem legte Sigonio dar, dass die kirchenhistoriographischen „Umstände“, in die er die Konstantinische Schenkung in seinem De occidentali Imperio einbettete, problematisch seien. Wie Lorenzo Valla vor ihm behauptete Sigonio, dass die legendäre Schenkung des spätantiken Kaisers vor seiner Heilung durch Papst Sylvester I. und seiner anschliessenden Bekehrung zum Christentum im Widerspruch zum Sieg gegen Maxentius unter dem Zeichen des Kreuzes gestanden haben musste. Dieser kirchenhistoriographische Widerspruch in einer Datierung der Schenkung stellte für Sigonio aber mehr ein theologisches Problem in Hinblick darauf dar, wie die Kaiserfigur angemessen darzustellen war: Entweder sollte Konstantin d. Gr. vor seiner Bekehrung zum Christentum als arianischer Verfolger früher Christen betrachtet werden oder man erwähnte nur die Taufe und Heilung des Kaisers durch Papst Silvester I. Sigonio bevorzugte letztere Deutung, denn in der ersten hätte er Silvesters Vorläufer, Papst Milthiades, als Märtyrer darstellen müssen. Die Konstantinische Schenkung war demgemäss nicht als kaiserlicher Gehorsam gegenüber dem Papsttum anzusehen. Vielmehr interpretierte sie Sigonio als Resultat eine Ehrerweisung, die der Kaiser gegenüber Papst Silvester I. als Dankesakt für seine Heilung geleistet habe. ${ }^{567}$ Ein weiteres Schriftstück aus der Bibltioteca Vaticana zeigt ferner, welche kirchenhistoriographische Bedeutung die Auseinandersetzungen der Kurie mit der Konstantinischen Schenkung zum Zeitpunkt von Sigonios De occidentali Imperio eingenommen haben musste. In dieser wahrscheinlich um 1577 verfassten Handschrift kann eine Änderung in der Formulierung der Begegnung zwischen Kaiser Konstantin d. Gr. und Papst Silvester I. beobachtet werden. Obwohl die angenommene Christenverfolgung des Kaisers immer noch unerwähnt blieb, wurde Konstantin d. Gr. vom Papst geheilt und getauft. Das Dokument fügte dieser Darstellung eine Zusammenfassung des edictum an, samt weiteren Zeichen der kaiserlichen „Wohlgefälligkeit“ (gratia). ${ }^{568}$ Sigonios Neuauslegung hatte die Konstantinische Schen-

566 BAM, P 193sup., f. 4r-8v; Sigonio, Opera omnia, VI, S. 985-988.

567 Sigonio, Opera omnia, VI, S. 987: „Quomodo ergo Constantinus anno 315 potuit esse Arrianus? Haec ejusmodi confinxerunt, qui temere voluerunt defendere omnes partes edicti, \& nullam historiam tenuerunt, inter quos Principatum habet Episcopus Eugubinus, qui maledicit potius, quam defendit. Quid igitur censes ne Ecclesiae Romanae auctoritas contemni videatur, \& ut repugnantia historiarum tollatur?“ Zum Umgang mit der Konstantinischen Schenkung nach Lorenzo Vallas Declamatio vgl. Setz 1975; Vian 2004, S. 129-168; Antonazzi 1985, S. 105-107; Malesevic 2015, S. 1214. Im Zusammenhang mit der posttridentinischen Konzeption der historia ecclesiastica dann insbes. Prodi 1977, S. 91, 96 und idem 2005, S. 298-307.

568 BAV, Vat. lat. 6160, f. 137v: „Ob id Constantinus e sancto lavacro lepra liber emersus, ut gratiam tanti beneficii Sylvestro referret, editum, quod nunc quoque legitur, scripsit, in quo, hac historia enarrata, adiicit se ad referendam gratiam, Sylvestrem omnibus ad amplitudinem ac dignitatem Apostolicae sedis ornandam insignibus decorare atque eumdem et successores eius ipso insuper 
kung aber immer noch nicht dem kurialen Verständnis, wie es vor allem die Indexkongregation in Sigonios De occidentali Imperio theologisch wiedererkennen wollte, entsprechend angepasst. Der Schenkungsakt sollte nämlich an sich als ein bewusster Akt der kaiserlichen Umkehr dargestellt werden. Konstantin d. Gr. wäre dadurch vom arianischen Christenverfolger zu einem Christenförderer übergetreten. Damit wäre dann auch die grundsätzliche Anfechtung des Constitutum vom eigentlichen Schenkungsakt getrennt. ${ }^{569}$

Trotzdem erhielt Carlo Sigonio vom Boncompagni-Papst die Erlaubnis, sein $D e$ occidentali Imperio drucken zu lassen. Diesen Umstand hatte er der Unterstützung des Kardinals und Erzbischofs von Bologna, Gabriele Paleotti, zu verdanken. Bereits 1565 traten Paleotti und Sigonio in eine enge Zusammenarbeit ein, die hauptsächlich aus Paleottis eigenen Interessen und der Förderung juristischer Gelehrtenkultur in seiner Diözese entstand. Paleotti hatte sich nach dem Druckverbot von Sigonios Historia Bonnoniensis in seinem Briefwechsel mit dem Kardinalsbibliothekar Sirleto um die päpstliche Druckerlaubnis für Sigonios De occidentali Imperio bemüht. ${ }^{570} \mathrm{Da}$ durch gelang es dem Bischof von Bologna, das Privileg für die Druckgenehmigung von Sigonios Werk in Bologna zu erhalten. Zum Zeitpunkt der Drucklegung von Sigonios De occidentali Imperio hatte der Papstneffe Giacomo Boncompagni, der auch das ducato von Cesare Baronios Heimatstadt Sora verwaltete, Sigonio nach Rom bestellt. Das unvollendete Projekt der Historia Ecclesiastica lässt sich aus einem Briefschreiben Sigonios an seinen Freund Alberto Bolognetti auf seinen letzten Romaufenthalt im Herbst des Jahres 1578 datieren. ${ }^{571}$ Im Februar 1579 sandte Sigonio die ersten drei Bände nach Rom. Diese sollte wieder der Präfekt der Indexkongregation Sirleto im Hinblick auf deren Drucklegung prüfen lassen. Ein Briefschreiben Sigonios an Sirle-

Occidente donare. Quod edictum Ecclesia Romana in hunc usquediem constantissime tenuit“. Vgl. hierzu dann auch McCuaig 1989, S. 260-261; Zen 2017, S. 18.

569 Malesevic 2015, S. 60-62.

570 Prodi 1959/67, Bd. 2, S. 256-257; McCuaig 1989, S. 260-262. Dies betrifft folgende, an Kardinal Sirleto adressierte Brieschreiben: BAV, Vat. lat. 6946, II, f. 369r; BAV, Vat. lat. 6416, f. 53r-v; ivi, Vat. lat. 6193, f. 109r; ivi, Vat. lat. 6181, f. 353r; ivi, Vat. lat. 6191, I, f. 226r. Sigonio hatte in der ersten Auflage dem Kardinal aus Kalabrien gedankt. Vgl. Sigonio, Historiarum de occidentali imperio, c. 70: „Quod Edictum Ecclesia Romana atque adeo etiam Orientalis in hunc usque diem constantissime tenuit, Theodoro Balsamone et Emanuele Caleca et Gennadio patriarcha, Graecis scriptoribus, testibus, quorum illustrissimus Cardinalis Sirletus opportune iam inde Roma usque admonuit“.

571 Vgl. das Schreiben Sigonios an Bolognetti vom 6. Dezember 1578 in Tiraboschi, Biblioteca Modenese, V, S. 92: „Tornai da Roma albergato dall'Eccellentissimo Signore Giacomo honoratamente. Hebbi un cavalleriato di S. Paolo et carico di scrivere l'Historia Ecclesiastica con molte promesse appresso“. Die handschriftliche Überlieferung des Briefes befindet sich in Biblioteca Estense, Alpha, G.1.18 (Ital. 835), Nr. 8. Vgl. auch das Briefschreiben Sirletos an Paleotti vom 23. Oktober 1578 in BAV, Vat. lat. 6946, f. 257r: „[...] et tale quale possi Sigonio riuscire in scrivere l'Historia che si desidera sopra le cose della Santa Chiesa. N.S. se haverà caro che si attenda con quella fatica, stodio et diligentia che una materia tanto grande richiede“. 
tos Sekretär Federico Ranaldi verrät, dass Sigonio meinte, er sei im Stande, eine Kirchengeschichte ohne Konsultation römischer Bibliotheken - und allen voran der Vaticana - verfassen zu können:

Si puo far gran profitto, se saro aiutato. Ma come Sua Signoria Illustrissima sa, sono a Bologna, et solo, et senza libri; et come bisognerebbero un migliaio. Mi par di poter scrivere infino ad Innocentio terzo senza bisogno della libraria a Roma, o almeno senza gran bisogno. Ma pero non senza il consiglio di Sua Signoria Illustrissima, et l'aiuto. Il che ella conoscerà meglio delle scritture che io mandero, che io non saprei dire. Pero se nelli tre libri mandati sarà cosa che non habbia veduta, et sia sicura, et d'importantia, V.S. me n'avisi. Ella n'havrà la sua mercede poi in cielo, et se potrò nulla, anche in terra. Mi vien detta da tutti che non faro cosa buona senza vedere le centurie. Puo essere. Ma le centurie sono scommunicate, né saprei come far ad haverle; né voglio spendere quel poco che io ho in libri heretici. Questo scrivo, accioché ella il communichi con Monsignore Illustrissimo, al qualo io bascio la mano devotamente. ${ }^{572}$

Sigonio hatte sich somit auf ein Quellenmaterial gestützt, das innerhalb der kurialen Autorität der Indexkongregation - zumindest im Zusammenhang mit der Konstantinischen Schenkung - stark umstritten war. Er konnte daher seine Historia Ecclesiastica nicht vor der Drucklegung seines De occidentali Imperio in Angriff genommen haben. Im Handschriftenregister der Vaticana ist eine anonym gebliebene Kritik an Sigonios Historia Ecclesiastica überliefert, die sich in den kurialen Umkreis Guglielmo Sirletos und der Indexkongregation einordnen lässt. Der anonyme Kritiker moniert die Widersprüchlichkeit von Sigonios theologischer Auslegung der Vita Constantini Eusebius` v. Caesarea, da diese von arianischen Charakterzügen geprägt gewesen sei:

Tutto questo ho voluto scrivere per dire liberamente quel che sento, tanto piu ch'el Padre Maestro del Sacro Palatio (Paolo Constabili) m'ha detto che in questa historia gl'è parso di vedere alcune contradittioni manifeste, et per questo advertiva che se dovesse considerare bene innanzi che si sottoscrivesse [...] Bisogna dunque ch'el Signor Sigonio scrivi questa historia di Costantino in modo che non ve sia repugnantia alcuna, ma che sia conforme, servando quel ricordo, primo ne medium, medio ne discrepet imum. Per far questo me par che non debbi attendere a tutto quel ch'ognuno ha scritto, et tanto manco a quel ch'ha detto Eusebia Cesariense, al qual stando s'ha a credere Constantino fu Ariano, essendo stato battizato, secondo egli dice nel fine de la sua vita, da Ariani, del che è stato redarguito da Georgio Abbate, da Theophane, da Zonara monacho, da Georgio Cedreno et d'altri, non solo di questa bugia, ma ancora di

572 BAV, Reg. lat. 2023, f. 320r. Die drei nach Rom gesandten Bücher Sigonios mussten vor dem 21. März desselben Jahres dort angekommen sein, da Sigonio Kardinal Farnese in einem auf dieses Datum datierten Schreiben darum bat, ein gutes Wort für seine historia ecclesiastica einzulegen: „Ho mandato tre libri dell'Historia Ecclesiastica a Roma. La prego a favorirli, se li leggerà et truoverà degni di favore; se non li leggerà, almeno difendermi contra una nuova calunia, la quale intendo che infino ad hora mi vien data, che in troppo poco tempo habbia assalito una impresa così difficile et importante. Percioché essi considerano il tempo solamente nel quale ho havuto la commissione da Nostro Signore, ma io riguardo quello della mia vita, il quale ho speso tutto in scrivere“. Zit. n. McCuaig 1989, S. 78. 
molte altre [...] tutta la Chiesa Greca l'ha per santo et celebra la sua festa ohn'anno nelli tanti di maggio, come dunque puo essere che sia stato Ariano? Quest'ho detto per che non si debbi dar credito ad Eusebio, il quale essendo stato Ariano et Signifer Arianorum, come dice San Gerolamo et la settina Synodo, et come si vede nelli suoi trattati, cerca di mostrare che quell'Imperadore Catholico sia stato di quella setta heretica, quale lui ha fatta condennare nel Concilio Niceno primo [...] Sopra quel che me domanda le replico che non se deve sottoscrivere a questo trattato se prima non si leva tutto quel che genera dubio, et scrupolo, et contradittioni al Decreto della Donatione di Constantino Magno, all'epistola di Papa Adriano, alla settima Synodo generale, Nicena seconda, et al Breviario Romano. ${ }^{573}$

McCuaig hat die Autorschaft dieses Briefes Federico Ranaldi - dem Sekretär Sirletos innerhalb der Indexkongregation - zugeschrieben. ${ }^{574}$ Die anonyme Kritik knüpfte inhaltlich an die bereits im Zusammenhang mit Sigonios De occidentali Imperio angesprochene theologische Streitigkeit an, wie die Christenverfolgungen Kaiser Konstantins d. Gr. kirchenhistoriographisch mit seiner Schenkung in Bezug stand und ausgelegt werden sollte. Der anonyme Autor scheint mit seiner Kritik allen voran den Vorsteher der Indexkongregation überzeugt zu haben. Der anonyme Kritiker von Sigonios Historia Ecclesiastica schlug vor, das hochproblematische Constitutum Constantini nicht einer philologischen Kritik zu unterziehen, denn dies habe bereits Lorenzo Valla geleistet. Stattdessen sollte das Constitutum hauptsächlich anhand des Briefes an Papst Hadrian I., anhand der Glaubensartikel des zweiten Konzils von Nicaea sowie anhand des neuen Breviarium bewertet werden. Zudem sollte die redaktionelle sowie inhaltliche Straffung von Sigonios Historia Ecclesiastica den kurialen Revisionsarbeiten, die der Maestro del Sacro Palazzo zu ausgewählten liturgischen officia bereits hervorbrachte, angepasst werden. Die Konstantinische Schenkung - und mit ihr auch die translatio imperii - war daher gemäss ihrer Rolle innerhalb der Entwicklung des römischen Ritus neu zu bewerten.

Der anonyme Verfasser bezeichnet das Constitutum als bugia. Mit dieser Kennzeichnung bewegt sich der Verfasser auf einer von der Indexkongregation selbst vorgezeichneten Grenzlinie, mit welcher die kirchenhistoriographische veritas von häretischen Unwahrheiten kontroverstheologisch getrennt wurde. Solange eine solche kontroverstheologische Trennung eingehalten wurde, konnte auch keine Kirchengeschichte als Gegenentwurf zu den Magdeburger Zenturien erfolgreich herausgegeben werden. Schon am 18. Dezember 1579 teilte Sigonio dem Oratorianer Cesare Baronio mit, dass er sein Projekt einer Historia Ecclesiastica fallen:

Io non voglio che procediamo fra noi, come si suole fra i più, con dissimulationi socratiche, abbassando noi medesimi, et inalzando l'amico per modo di creanza; ma parliamo alla reale. Io adunque stimo infinitamente la virtù, et bontà sua. Senta pure, come ella vuole di sé medesima; et se ella ha così fatta opinione di me, o vera, o falsa che sia, me ne rallegro molto. Perciocché questo è il premio delle fatiche nostre, per mio giudicio sufficientissimo, con tutto che

573 BAV, Vat. lat. 3455, f. 11r-v.

574 McCuaig 1989, S. 261-262. 
non ne seguitasse altro commodo, che la buona opinione. Perciocché stimo adunque oltremodo il giudicio di V.S., desidero, che ella si faccia dare in mio nome da Monsignor Illustrissimo Sirletti (sic!) quei miei tre libri dell'Historia Ecclesiastica, et gli legga. Et più si degni avisarmi in generale, et in particulare ciò che gli pare, che si debba in essi alterare per migliorarli. Et di questo gli terrò obbligo in eterno, pregandola a comandare anche a me, in che io spenderò volontieri ogni opera, et studio mio..$^{575}$

Cesare Baronio sollte daher vom Präfekten der Indexkongregation und Kardinalsbibliothekar Sirleto die Erlaubnis erhalten, die Historia Ecclesiastica Sigonios zu lesen und dieses Projekt weiterzuführen. Es ist aber möglich, dass sich hinter der vorhin erwähnten anonymen Kritik an Sigonios Historia Baronio selbst verborgen haben könnte. Die Bezeichnung des Constitutum Constantini als „Lüge“ (bugia) kommt nämlich in einem späteren Briefschreiben Baronios an Antonio Talpa wieder vor. ${ }^{576}$ Dies würde aber eine noch weit intensivere Einbindung Baronios in die nach 1572 in der Kurie eingerichtete Schreibwerkstatt gegen die Magdeburger Zenturien bedeuten. Im folgenden Kapitel dieser Untersuchung soll daher gründlicher auf Baronios Eintritt in den kurialen Herrschaftsapparat Gregors XIII. eingegangen werden.

575 ACN, XVII, f. 119r; Alberici, Epistolae et opuscula, III, S. 139. Jedoch hatte Sigonio bereits in einem Schreiben vom 4. April desselben Jahres an Fabio Albergati seine Gründe für das „Aufhalten“ seiner Historia Ecclesiastica genannt, da er sich nämlich einer Neuausgabe seiner Historia Bonnoniensis widmen wollte. Vgl. dazu McCuaig 1989, S. 79; Zen 2017, S. 19.

576 ACN, III, f. 2014r: „Fin qui il P. Tomaso non mi ha mostrato niente dei suoi scritti circa la dubitatione della donatione de Costantino della quale bastaria a lassarsi da banda, l'essersi trovato l'impostore autore di essa Giovanni Diacono. Il che se ben mancasse, non di meno il vedere che Costantino dividendo alli figlioli in tre parte l'imperio, et lassando Roma con l'Italia con altre provincie a costante. Che vogliamo dire? non altro se non che si fusse pentito della fatta donatione. Di più mai son mancati in Roma li prefetti messi dalli Imperatori, i quali gli pontefici portano ogni honore et ossequio. Di più mai si è mostrato atto alcuno de Pontefici Romani per spatio di settecento anni, se pur detta parola; che sia stata mai fatta detta donatione. [...] Di più l'editto della donatione è pieno di bugie inescusabili. Vi prego per carità non mi fatte imbrattar la penna a scrivere et defendere si fatte menzogne a Dio odibili, qual'è Dio di verità. Ho visto quanto sopra di cio è stato scritto da altri in difensione, et trovo tutto esser pagliaccia, ne credo possi aggiungervisi altro“. Das Briefschreiben ist auf den 1. Dezember 1590 datiert. Ein Briefschreiben Baronios an Sigonio konnte bislang aber noch nicht aufgefunden werden. 